PALEO

Revue d'archéologie préhistorique

21 | 2009-2010

Varia

\title{
La pointe de Font-Yves et les productions lithiques des derniers Aurignaciens
}

Damien Pesesse

\section{(2) OpenEdition}

1 Journals

Édition électronique

URL : http://journals.openedition.org/paleo/1823

DOI : $10.4000 /$ paleo.1823

ISSN : 2101-0420

Éditeur

SAMRA

\section{Édition imprimée}

Date de publication : 1 mars 2011

Pagination : 203-222

ISSN : 1145-3370

\section{Référence électronique}

Damien Pesesse, "La pointe de Font-Yves et les productions lithiques des derniers Aurignaciens »,

PALEO [En ligne], 21 | 2009-2010, mis en ligne le 21 octobre 2011, consulté le 07 juillet 2020. URL

http://journals.openedition.org/paleo/1823 ; DOI : https://doi.org/10.4000/paleo.1823

\section{(c) (i) () $\Theta$}

PALEO est mis à disposition selon les termes de la licence Creative Commons Attribution - Pas

d'Utilisation Commerciale - Pas de Modification 4.0 International. 


\title{
LA POINTE DE FONT-YVES et les productions lithiques des derniers Aurignaciens
}

\author{
Damien PESESSE ${ }^{(1)}$
}

\begin{abstract}
Résumé : En 1905, lors de la fouille de la Grotte de Font-Yves (Corrèze), un nouveau type de pointe en silex fut découvert : la pointe de Font-Yves. L'essentiel de l'assemblage se rapporte à une industrie d'“ ambiance » aurignacienne parmi laquelle au moins deux composantes peuvent être distinguées, l'une se rapportant à un Aurignacien ancien dominant et l'autre à l'Aurignacien récent. En dépit de la spécificité de cette industrie, l'intégrité de cette collection n'a paradoxalement jamais été discutée. Or, aucun lien technique ne peut être établi entre les pointes de Font-Yves et la composante aurignacienne classique.

Très rapidement, cette pointe originale fut comparée aux pointes de Krems et aux pointes d'El Wad. A partir de ces comparaisons et d'une suite de définitions pour le moins partielles, la pointe de Font-Yves réintégra la typologie française complètement dénaturée. Des spécimens attribués à ce nouveau type furent retrouvés dans plusieurs assemblages se rapportant aux débuts de l'Aurignacien, tels le Piage ou l'Arbreda. A l'heure actuelle, la pointe de Font-Yves serait, avec la grande lamelle Dufour, une des armatures réalisées par les toutes premières populations aurignaciennes. Or, entre ces nouvelles pointes de Font-Yves et les pièces éponymes de nombreuses différences apparaissent. De bien meilleures affinités peuvent être mises en évidence entre les pièces éponymes et les spécimens présents dans la couche 6 de l'Abri Pataud. Dans ce sens, la pointe de Font-Yves ne peut plus être considérée comme un marqueur des phases initiales de l'Aurignacien mais au contraire comme l'une des dernières innovations en matière d'équipement lithique des populations aurignaciennes.
\end{abstract}

Mots-clés : Aurignacien, système technique, armature, attribution culturelle, transition.

Key-words: Aurignacian, technical system, dart, cultural attribution, transition.

\section{Abriged english version}

\section{The Font-Yves point and the lithic productions of the last aurignacians.}

During the excavation of the Grotte de Font-Yves, a new kind of flint artefact was discovered : the Font-Yves point. The Grotte de Font-Yves industry shows numerous Aurignacian characters (fig. 1, 2). The major part of this industry can be related to an early Aurignacian, but some pieces belong to a recent Aurignacian. Despite the specificity of this industry, its integrity has never been questioned. Indeed, there is no link between the Font-Yves points (fig. 3 to 5) and the classsical Aurignacian component. Early on, the Font-Yves point was compared with Krems points and El Wad points (fig. 7, 8). Based on those comparisons and many incomplete definitions, the Font-Yves point was inappropriately integrated into French typology. Artefacts attributed to this new type have been found in archeological assemblages related to the early Aurignacian, like Le Piage and Arbreda. Today, the Font-Yves point, along with long Dufour bladelets, is considered as a lithic armature made by the very first Aurignacian populations. But many differences exist between those «new Font-Yves points » and the eponymous points. Lithic points from layer 6 of Abri Pataud share more reliable technical characteristics with the point found in the Grotte de Font-Yves (fig. 10, 11). Therefore, the Font-Yves point can no longer be considered as a marker of the initial stage of the Aurignacian, but to the contrary, as one of the last innovations in the lithic toolkit of Aurignacian populations. 
La pointe de Font-Yves est classiquement considérée comme l'un des principaux marqueurs, avec la grande lamelle Dufour, des industries réalisées par les toutes premières populations aurignaciennes. Cette pointe serait présente sur l'ensemble de l'aire d'extension du premier Aurignacien en Europe occidentale, en Europe centrale où elle est fréquemment rapprochée de la pointe de Krems (Hahn 1977), puis aux marges orientales du peuplement aurignacien où elle est comparée à la pointe d'El Wad (Garrod 1934 ; Otte et al. 2007) ou d'Arjeneh (Otte, Kozlowski 2009). Cette pointe participerait au développement d'un outillage leptolithique en liaison avec de profondes modifications des systèmes de production et vraisemblablement d'armement, communément imputées à l'arrivée de populations d'Hommes anatomiquement modernes.

A la lueur d'une réévaluation du mobilier de la Grotte de Font-Yves, plusieurs aspects du mode de production et de façonnage de la pointe éponyme peuvent être proposés. Sur ces bases, d'importantes différences apparaissent alors entre les pointes éponymes et les pointes rapportées par la suite à ce type. Nous essaierons donc de comprendre comment s'est effectué ce glissement sémantique et à quelle phase du Paléolithique supérieur nous pouvons rapporter ces pointes particulières. A l'heure actuelle, les meilleurs points de comparaison apparaissent avec les pointes de la couche 6 de l'Abri Pataud, c'est-à-dire à la fin de l'Aurignacien. Les implications de ce constat dépassent largement le cadre taxonomique. Ils permettent d'éclairer les processus de mutations culturelles paléolithiques dans le cadre du passage de l'Aurignacien au Gravettien dans le sud-ouest de la France.

\section{La Grotte de Font-Yves}

La grotte de Font-Yves s'ouvre au sud du plateau de Bassaler, dans un versant drainé par la Planchetorte (Bouyssonie et al. 1913 ; Bardon et al. 1920). Font-Yves se situe à un peu plus d'une dizaine de mètres de la FontRobert. Ces cavités correspondent à des abris peu profonds prolongés par une terrasse. Elles sont creusées dans les grès permo-triasiques du Bassin de Brive, par des phénomènes largement attribués à des alternances de gel / dégel (Raynal 1975).

Les abbés Jean et Amédée Bouyssonie ainsi que Louis Bardon publient peu d'indications sur la fouille même du gisement, ni la date précise des travaux, qui auraient eu lieu durant les années 1905-1906 (Demars 1994). La Comtesse de Thévenard aurait conduit les opérations de fouille. Les indications stratigraphiques révèlent la présence d'un niveau d'effondrement de la voûte qui scella l'unique niveau archéologique, constitué de terre noirâtre, en légère pente vers la vallée. Une seule couche archéologique a été reconnue. Elle est attribuée à l'Aurignacien, bien que des fragments de pointes de la Gravette, des indices de Solutréen, ainsi que des débris de poterie aient été trouvés (Bouyssonie et al. 1913). Yves, le fils de la Comtesse de Thévenard, serait le principal acteur des fouilles (Movius 1980).
En 1979, G. Mazière entreprit une opération de terrain dans la cavité. Plusieurs sondages, représentant une surface de $7 \mathrm{~m}^{2}$, ont été réalisés par J.-P. Chennbaut (P.-Y. Demars, comm. pers.) : aucun niveau archéologique n'a été retrouvé (Mazière 1980). Le tamisage des anciens déblais a permis toutefois de recueillir une quantité importante de mobilier lithique.

\section{Historique des attributions}

D'un point de vue historiographique, ce gisement tient une place particulière. Face à la spécificité de l'industrie, beaucoup d'opinions divergentes furent avancées.

Les abbés ont clairement énoncé les difficultés d'un calage chronologique (Bouyssonie et al. 1913 ; Bardon et al. 1920). Si une part du matériel rappelle fortement le niveau inférieur de la Coumba del Bouïtou, leur discours laisse place à certaines ouvertures liées à la nature " embarrassante » de l'industrie (Lalanne, Bouyssonie 1946 - p. 87). Toutefois, c'est essentiellement le parallèle avec ce gisement et d'autres contemporains qui fut retenu. Le matériel présente effectivement de nettes affinités avec des assemblages relevant de l'Aurignacien ancien classique : lames aurignaciennes, lames étranglées, grattoirs carénés, grattoirs à museaux. Au sein de l'outillage, certains aspects typologiques et stylistiques nuancent toutefois une attribution pleine et entière à l'Aurignacien ancien (Bouyssonie et al. 1913 ; Bardon et al. 1920), par l'importance numérique des burins et le fait que les éclats et les lames « $n$ 'ont pas, à quelques exceptions près, la belle venue des lames retouchées du Bouitou » (Bardon et al. 1920 - p. 295). Leur conclusion révèle clairement ce questionnement chronologique:

« la Font-Yves présente d'une part une ressemblance extraordinaire avec le Bouitou inférieur, mais elle est loin d'être aussi riche ; d'autre part, ses lames à bord abattu tendent à l'éloigner de ce niveau, à la rajeunir même plus que le Bouitou supérieur. Peut-être, l'habitat de cette station a duré assez longtemps et a vu la transformation de l'Aurignacien ; peut-être y a-t-il eu simple survivance de types anciens ? » (Bouyssonie et al. 1913 - p. 225).

Tous les auteurs ultérieurs ne firent pas preuve d'une telle prudence, en particulier D. de Sonneville-Bordes (1958 p. 446) : "Tous les outils caractéristiques de l'Aurignacien (grattoirs carénés et à museau, lames aurignaciennes, lames étranglées) sont présents à Font-Yves et les proportions des divers types d'outils représentés sont les mêmes que pour les outillages du Périgord, dont l'attribution à l'Aurignacien typique est indiscutable. "

Denis Peyrony, dans la seconde phase de sa théorie, place cette industrie dans la lignée phylétique du Périgordien II (Peyrony 1946).

Le fort indice de burin fut à l'origine de rapprochements avec des séries se rapportant à l'Aurignacien récent lato sensu. G. Laplace établit des comparaisons avec la Ferrassie couches H, H', H”, Cellier couche G, la Faurélie, 
les Vachons couche 2, les Cottés couche D, Chanlat foyers supérieurs et Willendorf couche 4 (Laplace 1966). Cette industrie aurignacienne, par la présence d'une composante lamellaire à dos, constituerait un substrat potentiel à la formation du Gravettien (Laplace 1966). G. Mazière reprend cette comparaison avec La Ferrassie, niveaux Gos et Gf des fouilles H. Delporte. II statue en faveur d'« un Aurignacien très évolué, Aurignacien III voire IV (?) 》 (Mazière 1978). Pierre-Yves Demars conclut à un moment bref et localisé de l'Aurignacien Ic (soit Aurignacien ancien de type Ferrassie, Demars 1992), interprétant la pointe de Font Yves comme " une variété probablement éphémère " de la lamelle Dufour (Demars 1994 - p. 98). Plus récemment, la présence des pointes lithiques a également orienté l'attribution globale de la série vers une phase initiale de l'Aurignacien (Bon 2000, 2002).

Aurignacien archaïque, Aurignacien ancien, Aurignacien récent, Aurignacien très évolué, toutes les attributions possibles au cours de l'Aurignacien ont été proposées. L'importance du groupe des burins a souvent été prise en compte, sans pour autant interroger la nature des burins présents.

Toutefois, la série de Font-Yves ne se limite pas aux pièces que nous venons d'évoquer et la présence de cinq pointes de La Gravette, de fragments de pointes à cran solutréennes n'a jamais jusqu'à présent été introduite dans la discussion. Plus particulièrement, la co-existence d'un fonds classique aurignacien avec ces pointes de FontYves originales a toujours été perçue comme une donnée implicitement admise. La nature même d'une telle association n'a jamais été véritablement questionnée. L'industrie fût considérée comme " homogène ", (Mazière 1978 ; Mazière, Tixier 1976), « parfaitement homogène » (Movius 1980).

\section{Le matériel}

La collection étudiée, la collection Thévenard, est conservée au dépôt archéologique de Chasteaux (Corrèze). La série déposée dans les réserves du Musée Labenche a également pu être examinée. L'assemblage se compose essentiellement de supports retouchés, relativement nombreux. Les premiers auteurs dénombrent à peu près 925 outils et débris d'outils (Bouyssonie et al. 1913 ; Bardon et al. 1920). Denise de Sonneville-Bordes décompte 1237 outils (Sonneville-Bordes 1960), 1256 pour G. Laplace (1966). G. Mazière retient 983 pièces (Mazière 1978) et P.-Y. Demars 1056 outils (Demars 1994).

L'essentiel de la fraction brute et de la fraction lamellaire est absent. Elle a d'ailleurs été retrouvée en quantité importante lors du sondage de G. Mazière (1980). Nous disposons donc seulement des produits retouchés, souvent entiers, de dimensions plutôt importantes et des nucléus. Le nombre de nucléus, la diversité apparente des objectifs et l'absence de fraction lamellaire, particulièrement la plus fine, rend toute attribution de l'ensemble relativement arbitraire. En revanche, les principales tendances peuvent être distinguées et décrites (Pesesse 2008a).
Une première composante laminaire s'individualise (fig. 1). Elle correspond à un débitage unipolaire de belle facture, au sein duquel la percussion organique prédomine. Ces supports sont transformés en grattoirs, lames retouchées, burins. Aucun nucléus ne se rapporte à cette production.

Parmi les nucléus, plusieurs appartiennent à une production de supports rectilignes légers que nous décrirons plus loin. De nombreuses pièces carénées sont présentes (fig. 2). Elles se rapportent à des productions de type grattoir, grattoir à museau, burin caréné, burin des Vachons ou réalisées à partir d'un pan de la face supérieure de l'éclat nucléus.

Les productions répondent donc à des intentions distinctes en termes de gabarits et de paramètres morphologiques. Cette diversité concerne aussi bien les objectifs que les modalités. Si une part des débitages suit des modalités inédites, de nombreux éléments techniques recoupent des intentions clairement définies dans des contextes aurignaciens (Chiotti 1999, 2003 ; Lucas 2000 ; Bon 2000, 2002 ; Bordes 2005).

Cela corrobore les observations des abbés et les comparaisons avec différents stades de l'Aurignacien à partir de la séquence de la Coumba del Bouïtou. Une forte composante attribuable à l'Aurignacien ancien est présente, mais d'autres éléments notamment des productions lamellaires sur tranche d'éclat attestent d'une ou de plusieurs occupations datant d'une phase plus récente de l'Aurignacien.

Les pointes de Font-Yves et les éléments techniques associés (nucléus, rares sous-produits) se trouvent fortement isolés dans cet assemblage. En effet, cette composante n'est pas compatible techniquement avec un Aurignacien classique et doit donc être décrite séparément. Face à la spécificité de ces pointes et la typicité d'une large part de l'industrie, l'absence de remise en question de l'intégrité de cet ensemble paraît étonnante.

\section{Les pointes de Font-Yves}

Certaines tendances ou principes opératoires de la production des pointes de Font-Yves peuvent être mis en lumière, en dépit de l'ancienneté de la collection. Ces éléments techniques possèdent un caractère diagnostique et pourront servir de critères de discrimination dans le cadre de comparaisons. La pointe de Font-Yves est un type spécifique, dont l'homogénéité et la standardisation constituent les principales caractéristiques (fig. 3).

\section{Les pointes}

D'après P.-Y. Demars, la composition pétrographique du corpus de pointes diffère du reste de l'assemblage. Ceci constitue un argument supplémentaire pour dissocier ces artefacts de l'ensemble de l'industrie. Les pointes sont essentiellement en silex du Sénonien $(n=52) ; 11$ sont en Bergeracois, le reste, en silex de l'Hettangien et du 

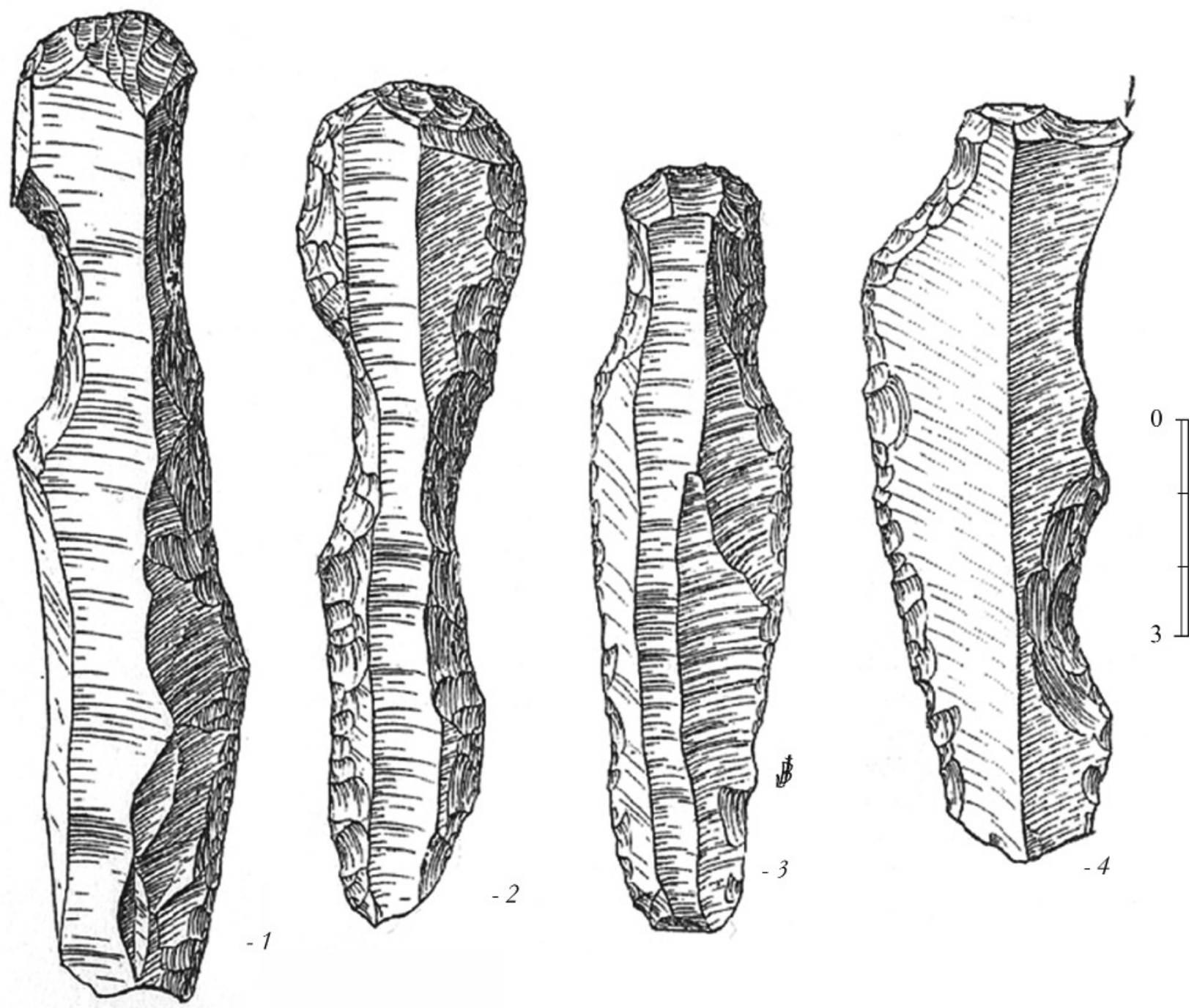

Figure 1 - Grotte de Font-Yves : Lames (dessins J. Bouyssonie).

Figure 1 - Grotte de Font-Yves : Blades (drawings J. Bouyssonie).

Cénozoïque (Demars 1994 - p. 101). Elles sont au nombre de 67 , sur 140 décomptées initialement par J. Bouyssonie 2 . Les fragments proximaux dominent $(n=30)$, les mésiaux sont peu représentés $(n=4)$, alors qu'il existe 17 fragments distaux, pour 8 pointes sub-entières et 3 entières seulement.

Les exemplaires complets mesurent entre 32 et $70 \mathrm{~mm}$ de long, paramètre vraisemblablement plus étendu si l'on prend en compte la longueur de la plus petite pièce subentière : $23 \mathrm{~mm}$. Les gabarits sont donc très différents, cependant les proportions relatives s'avèrent stables. Au moins deux pièces se distinguent par leur sveltesse, qui évoque une production sur tranche d'éclat. Les pointes mesurent de 5 à $10 \mathrm{~mm}$ de large pour 1 à $3 \mathrm{~mm}$ d'épaisseur (tabl. 1).

Mise à part la variation de module, aucun élément notable de diversité n'apparaît. Ces pointes sont très homogènes dans leur morphologie, si l'on considère leur élancement, leur rectitude et leur régularité. La principale source de variation concerne la latéralisation et l'intensité de la retouche. Celle-ci est toujours directe, fréquemment bilatérale. Hormis la base et l'apex, elle investit de manière variable les bords et aucune partie latérale n'est systématiquement retouchée. Pour 65 cas diagnostiques :

- 4 fragments ont le bord gauche plus retouché que le droit, - 20 fragments ont subi un aménagement équivalent des deux bords,

- 41 fragments voient l'aménagement préférentiel du bord droit.

(2) Une partie des pointes, conservée dans les vitrines du Musée Labenche, n’a pas été prise en compte. 

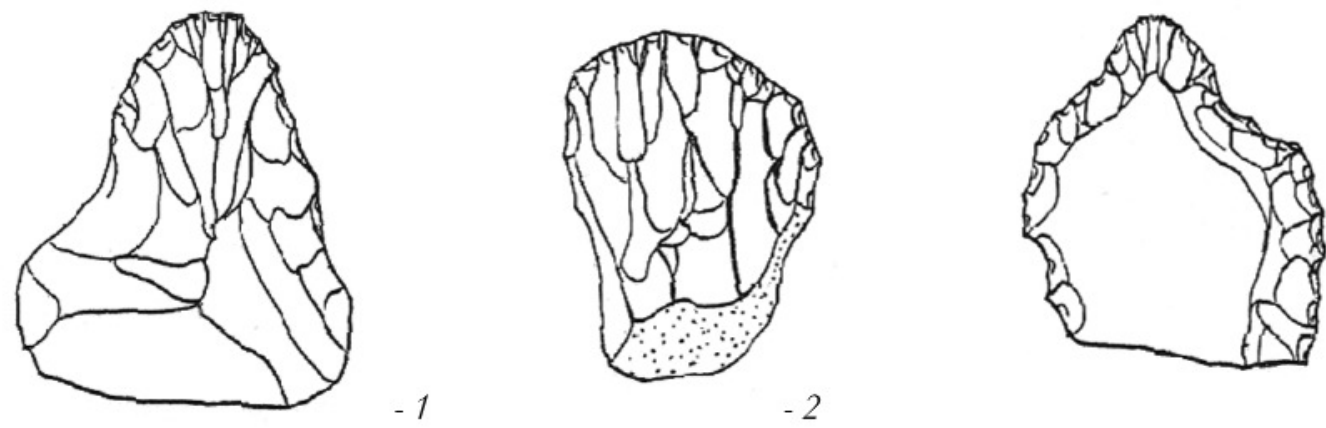

$-3$
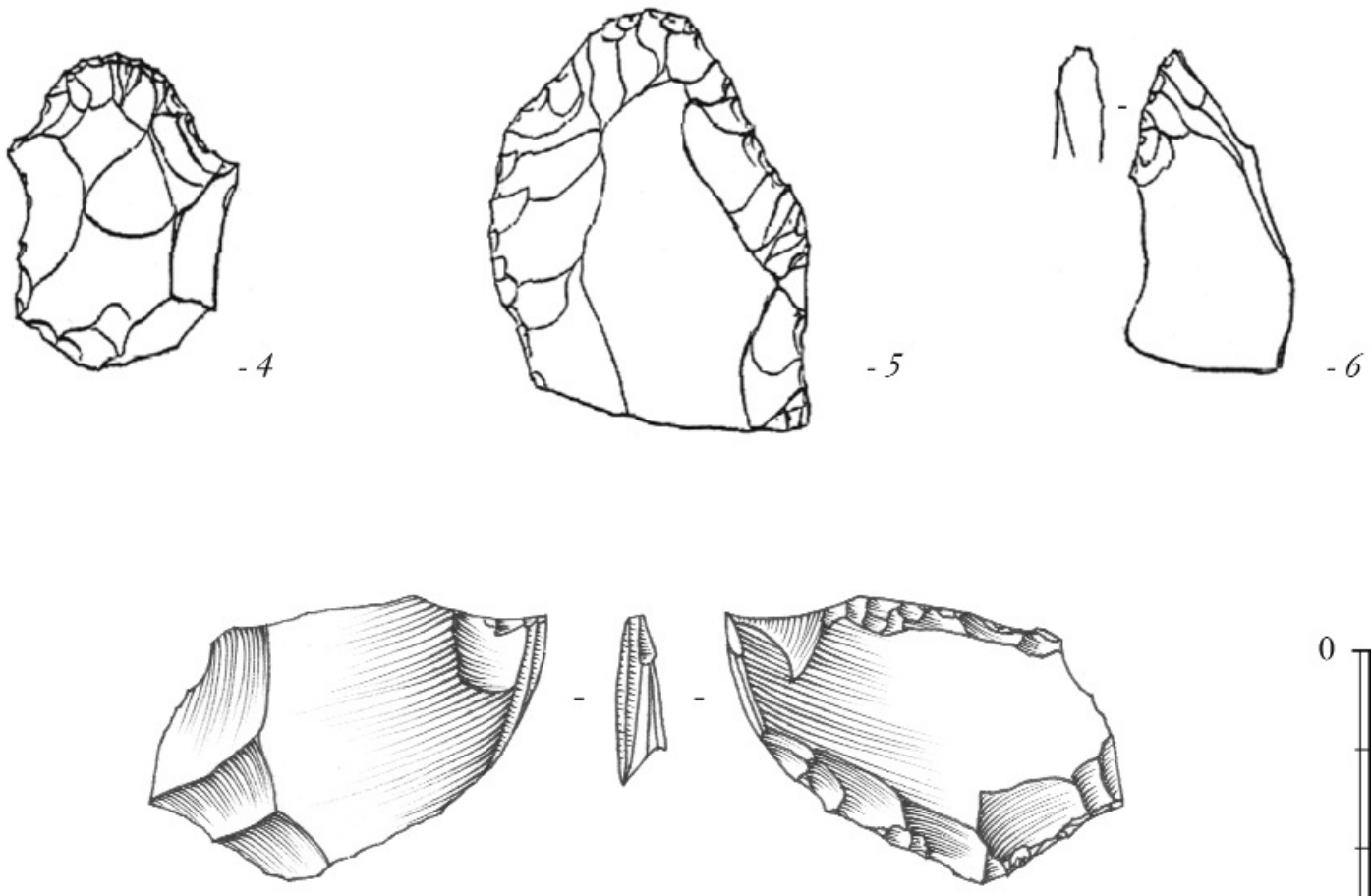

$-7$
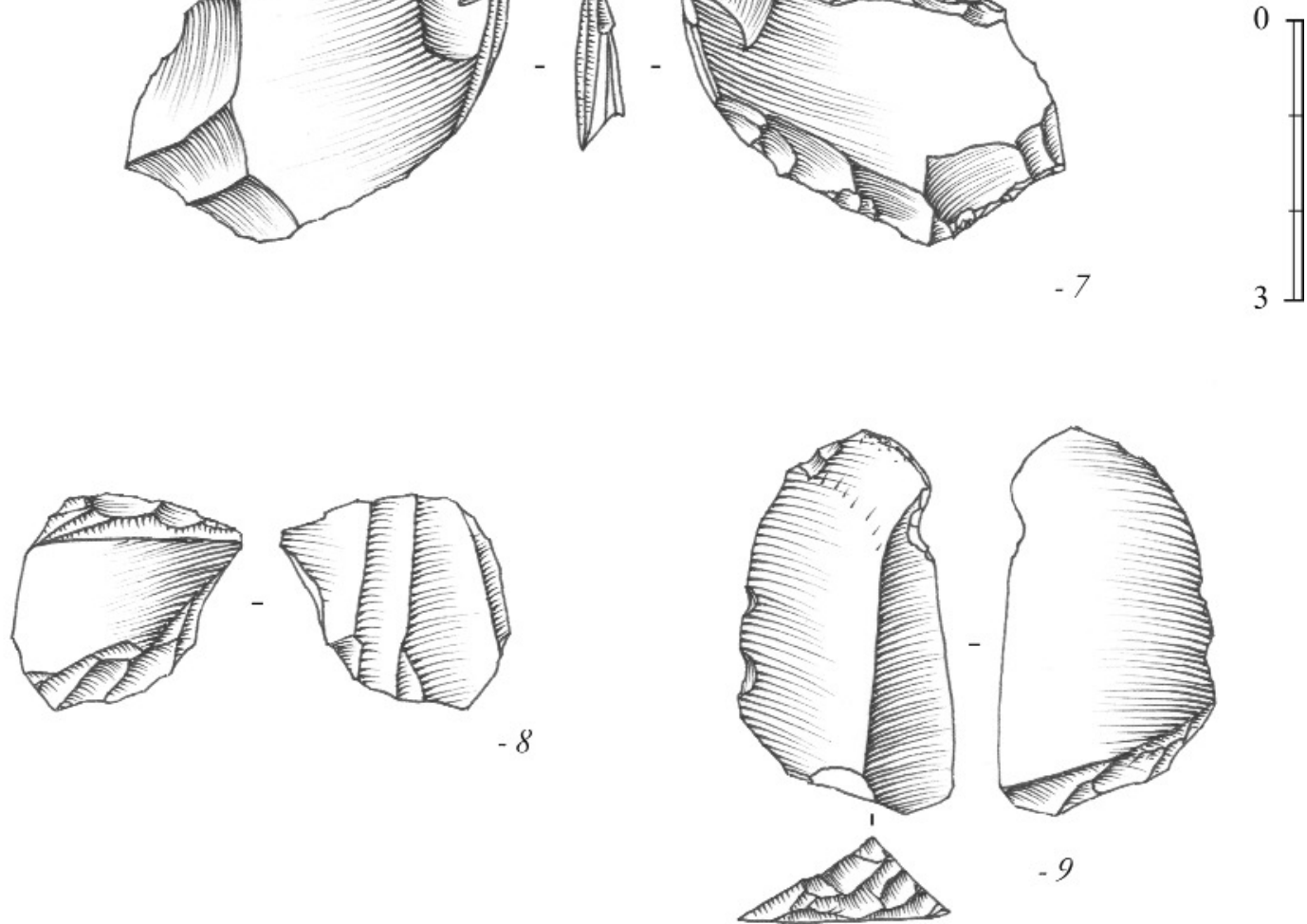

Figure 2 - Grotte de Font-Yves : Nucléus à lamelles (dessins $n^{\circ} 1$ à $6: J$. Bouyssonie ; $n^{\circ} 7$ à $9: D$. Pesesse).

Figure 2 - Grotte de Font-Yves : Bladelets cores (drawings $n^{\circ} 1$ to $6: \mathrm{J}$. Bouyssonie ; $n^{\circ} 7$ to $9: D$. Pesesse). 

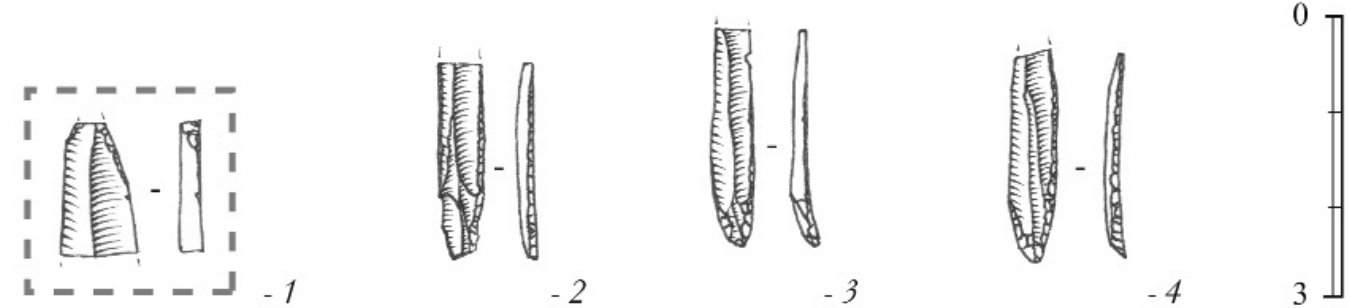

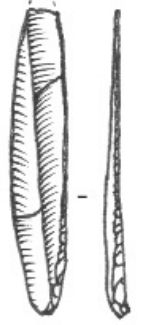

$-5$
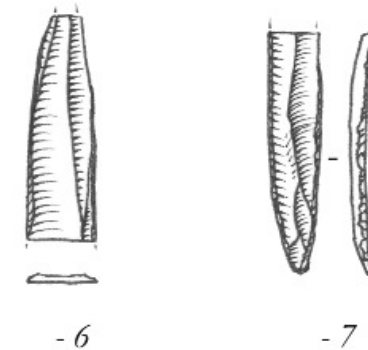

$-7$
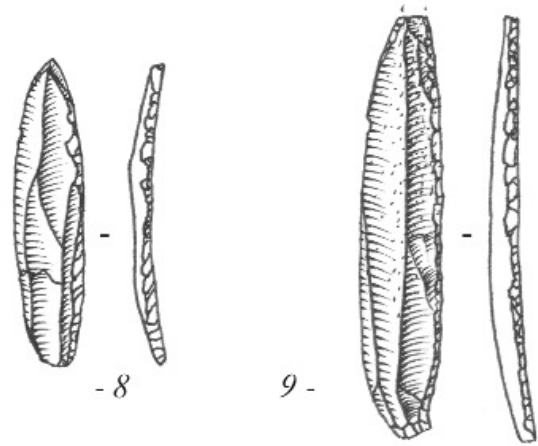

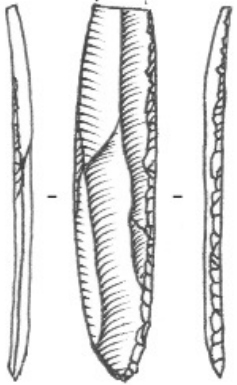

$-10$

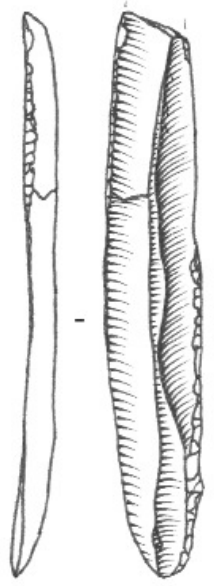

$-15$

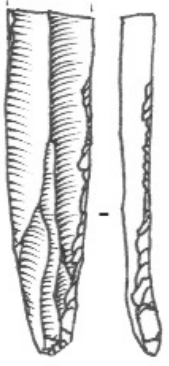

$-11$

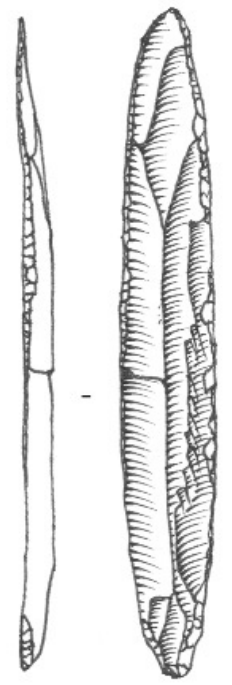

$-16$

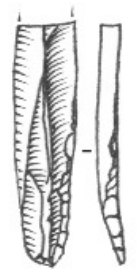

$-12$

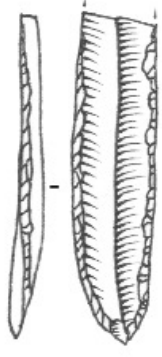

$-13$
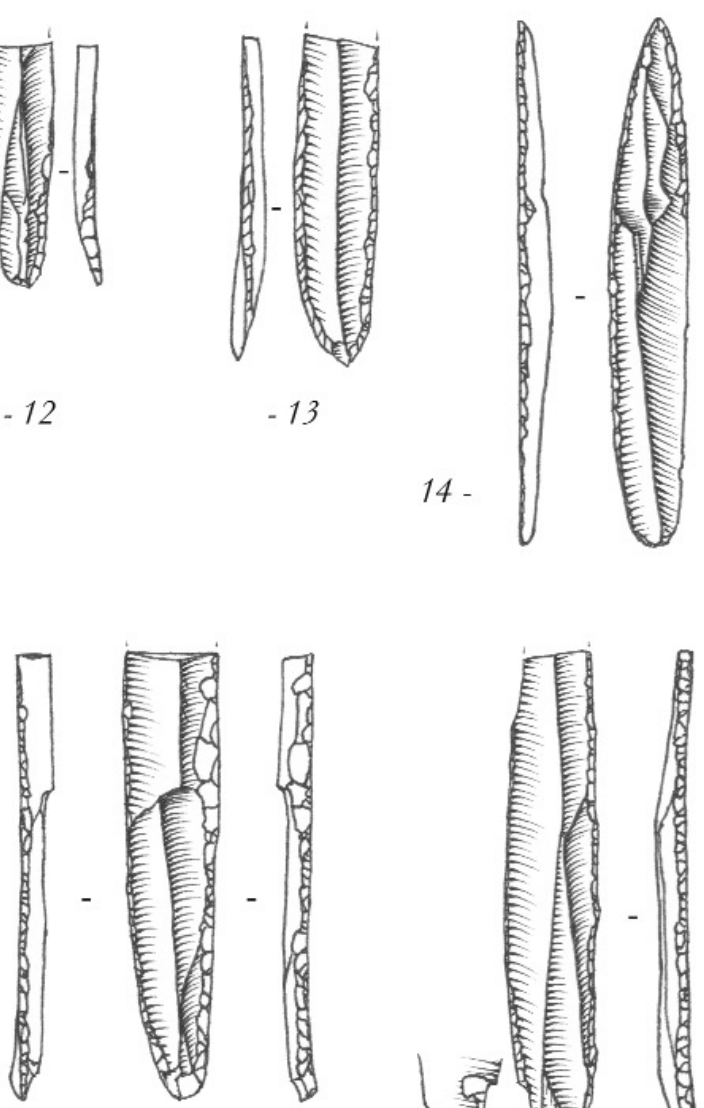

Figure 3 - Grotte de Font-Yves : Pointes de Font-Yves ( $n^{\circ} 1$ : fouille G. Mazière) (dessins D. Pesesse).

Figure 3 - Grotte de Font-Yves : Font-Yves points ( $n^{\circ} 1$ : G. Mazière's excavations) (drawings D. Pesesse). 


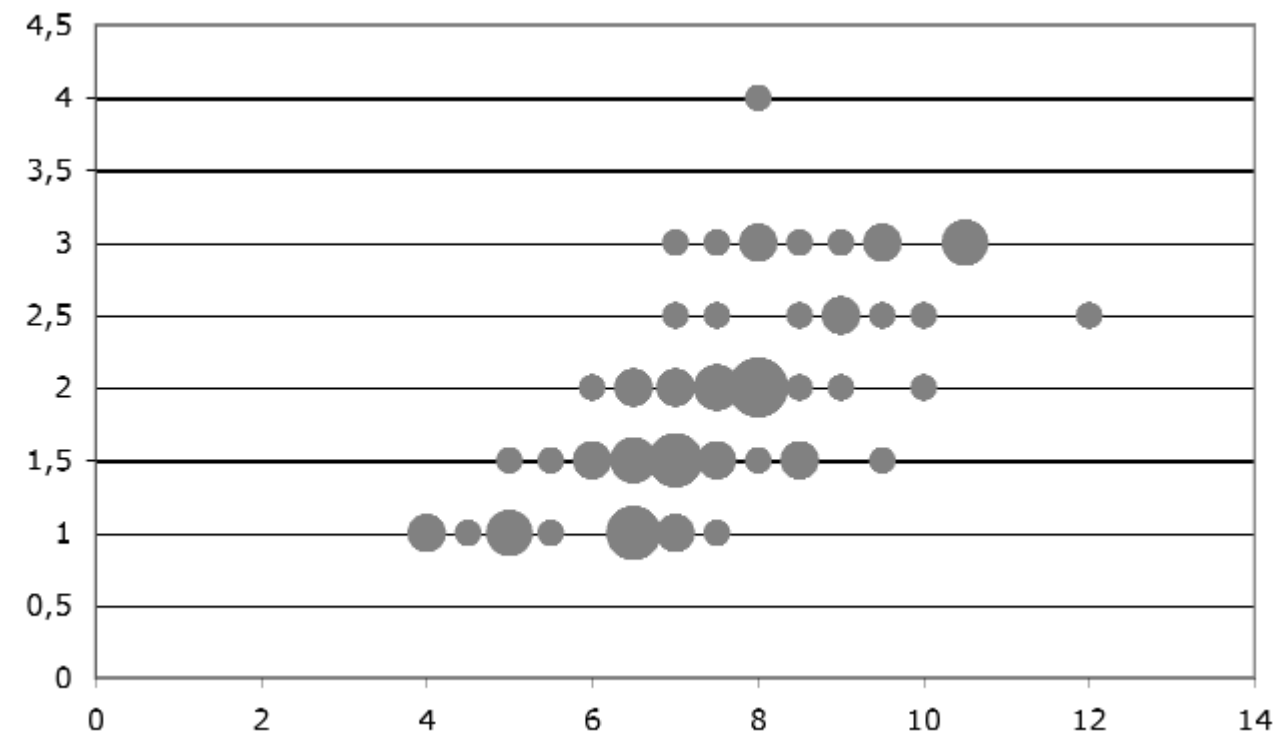

Tableau 1 - Rapport largeur/épaisseur des pointes de Font-Yves.

Table 1 - Ratio width/thickness of the Font-Yves points.

Si un nombre important de pointes n'est pas latéralisé, une latéralisation fréquemment dextre est observable.

La base est de morphologie majoritairement ogivale. Celle d'un unique exemplaire est tronquée. L'extrémité distale n'est pas particulièrement aiguë. Le terme de mousse, parfois employé (Pradel 1978), réduit tout de même le caractère potentiellement vulnérant de la pièce. La partie distale est mince, définie de manière axiale. Son aménagement présente une certaine variabilité, des extrémités brutes naturellement perforantes à des aménagements appointants. Elle est souvent de forme légèrement ogivale.

La retouche est marginale à semi-abrupte, pouvant devenir abrupte sur une zone épaisse. Son intensité est liée à la délinéation des bords du support. Globalement, la largeur de la pièce est peu réduite par la retouche. La transformation ne contribue pas spécifiquement à augmenter l'acuité des tranchants. Les bords définis sont parallèles, les extrémités axiales.

Sur certains spécimens, la retouche présente une caractéristique originale. Son axe n'est pas perpendiculaire à la face inférieure de l'outil, mais incliné d'environ $75^{\circ}$. L'inclinaison intéresse tout ou un segment du bord. Les pièces étant latéralisées, elle est surtout visible sur le bord droit. L'inclinaison est orientée vers la partie proximale pour le bord droit et la partie distale pour le bord gauche. Un geste de compression effectué à l'aide d'un épois en maintenant la pièce inclinée peut conférer une telle incidence à la retouche.
Aucun stigmate relatif à une utilisation en tant que projectile n'a été reconnu (O’Farrell 2004).

Les supports sélectionnés sont tous rectilignes, très peu présentent une légère concavité médiane ou une courbure distale. La seule courbure concerne la partie proximale de la pièce. Elle peut être imputée à la préparation au détachement (abrasion) qui concerne 30 des 42 parties proximales.

\section{Définitions}

La première description de la pointe de Font-Yves recoupe les critères que nous venons d'évoquer. L. Bardon, J. et A. Bouyssonie la définissent par rapport à celle de La Gravette (dénommée " canif ») : "la série des longues lamelles a une physionomie bien spéciale : les lames sont minces, très délicates ; l'écrasement ou rabattage du bord est moins brutal, moins abrupt ; le plus souvent les deux bords sont atteints par la retouche ; la section du couteau et du canif est un triangle, celle de ces outils rappelle plutôt un segment de cercle. Enfin, l'extrémité n'est pas toujours appointie, ni tronquée non plus « (Bouyssonie et al. 1913 p. 222).

Cette définition fut reprise par la suite (Lalanne, Bouyssonie 1946). Le caractère mousse de ces pointes a parfois été pris en compte (Pradel 1952, 1978 ; Mazière 1978). G. Laplace devant leur faible aspect perforant, et en comparaison avec les lamelles Dufour, suggère de nommer ces pièces « lamelles de Font-Yves » (Laplace 1961 - p. 170), 
appellation qui ne connut pas un réel succès. C'est pourtant ainsi que cette pointe est indexée dans certaines typologies (Demars, Laurent 1989).

La présence de supports acuminés tend à rejeter la proposition de G. Laplace. Si la perception du caractère vulnérant diffère de ses standards, elle ne doit pas être écartée a priori, à l'encontre des paramètres morphologiques. La pointe de Font-Yves peut être définie comme un outil sur support léger, rectiligne dont les bords sont rendus parallèles par une retouche de circonstance, souvent dextre, définissant une base ogivale et un apex axial dont l'aménagement varie dans son caractère appointant.

\section{Les nucléus}

Un minimum de 29 nucléus correspondant à une production rectiligne peut être distingué (fig. 4 ; fig. 5). II s'agit de 18 nucléus unipolaires (dont 7 sur éclats), 7 nucléus bipolaires (dont 5 préférentiels et 2 indéterminés), ainsi que 3 à plans de frappe opposés décalés et un à plans de frappe alternes. Ces nucléus sont essentiellement réalisés sur blocs.

Les nucléus font l'objet d'une mise en forme importante. Le dos est fréquemment aménagé, des crêtes postérolatérales sont présentes, les flancs préparés : le volume est parfaitement délimité. Parmi ces solutions, une option technique spécifique permet de regrouper certains nucléus. II s'agit de nucléus unipolaires dont la structure volumétrique renvoie principalement à une recherche de rectitude et de convergence des enlèvements. Cet aspect est autorisé par le resserrement basal de la table, lié notamment à l'extraction de supports envahissant largement les flancs, extraits depuis la base du nucléus. La quasi-absence de fraction brute correspondant à ce gabarit n'autorise pas à aller plus loin dans la compréhension des logiques opératoires. Signalons qu'un sous-produit, se rapportant à une reprise opposée latérale, est parfaitement compatible avec cette logique (fig. $5, n^{\circ} 3$ ).

Ce schéma unipolaire convergent, délimité par les flancs depuis la base du nucléus, constitue la modalité de production sur bloc la plus spécifique, mais d'autres solutions alternatives peuvent avoir contribué à la production de pointes. La série éponyme ne permet pas d'aller plus en avant dans la caractérisation des procédés de débitage dans leurs logiques propres et leur diversité potentielle.

\section{Le laminaire}

L'ensemble laminaire, en dépit d'une cohérence apparente, ne peut toutefois être rapporté exclusivement à l'une des occupations de l'abri. En effet, trente lames portent des négatifs lamellaires marqués (fig. 6), aspect encore inédit dans les productions laminaires de l'Aurignacien ancien ${ }^{3}$. Parmi ces supports, 20 sont transformés en grattoirs, 3 en burins, 4 sont retouchés, une lame est " appointée » et 2 demeurent brutes. On compte 11 lames réalisées en silex du Bergeracois, 15 en silex sénonien, une proviendrait du bassin d'Aurillac et 4 sont indéterminées.

Aucune lame indubitablement aurignacienne, d'un point de vue typologique (lame à retouche aurignacienne, lame étranglée) ne porte de négatif lamellaire marqué. Seule la lame « appointée » pourrait stylistiquement se rapprocher de l'outillage aurignacien.

Ces négatifs, rares et isolés, trop longs et trop réguliers pour être interprétés comme la conséquence de l'abrasion, évoquent des supports potentiels de pointes de Font-Yves. Si aucun argument ne permet de vérifier pleinement ce point, ce procédé, non documenté durant l'Aurignacien ancien, permet a minima d'envisager l'existence de plusieurs composantes techniques au sein du corpus laminaire.

\section{Le glissement}

La pointe de Font-Yves est reconnue dans de très nombreux gisements, ou plutôt des pointes à retouches bilatérales interprétées comme des pointes de Font-Yves sont mentionnées sur un vaste territoire. La répartition de ces pointes s'étend en effet de la Péninsule ibérique à l'Iran, où elles sont comparées aux pointes d'Arjeneh (Otte et al. 2007). Dans ces gisements, elle est associée à de grandes lamelles Dufour à tendance rectiligne et constitue un des éléments de définition du Protoaurignacien.

Or, ces pointes présentent de nombreuses différences avec les pièces éponymes (fig. $7, n^{\circ} 5$ à 14). En premier lieu, l'allure même diffère. Ces pièces sont réalisées sur des supports bien moins standardisés. La retouche bilatérale directe abrupte vise à aménager une extrémité axiale aiguë. Des pointes comme celles provenant du Piage (Champagne, Espitalié 1981 ; Bordes 2002) ne trouvent aucun écho dans la série éponyme. Au-delà des aspects fonctionnels, la norme qui préside à l'aménagement de ces pointes diffère. Il convient donc de comprendre comment s'est opéré ce glissement terminologique.

En 1912, l'Abbé Breuil dans sa première édition des «Subdivisions du Paléolithique supérieur » évoque ces pointes, avant même leur publication (Breuil 1912). II rapproche les pointes de Krems d' " une phase beaucoup plus primitive de l'Aurignacien moyen ; ses nombreux petits microlithes sont semblables à ceux, inédits encore » provenant de la Grotte de Font-Yves (Breuil 1912). Par la suite, il rapproche également Bos-del-Ser de ces deux sites (Breuil 1937).

La comparaison de Krems et Bos-del-Ser n'est pas sans pertinence. Cependant, cela instaure un premier lien entre ces industries et Font-Yves. Cette comparaison est reprise à l'identique par D. Peyrony qui associe le troisième foyer de la Grotte des Enfants (Peyrony 1946 - p. 234).

(3) Cet aspect a permis antérieurement à François Bon, Jean-Guillaume Bordes et Jacques Pélegrin de réfuter l'attribution de l'ensemble de la fraction laminaire à l'Aurignacien ancien, alors que d'autres éléments techniques s'y rapportent largement (comm. pers.). 

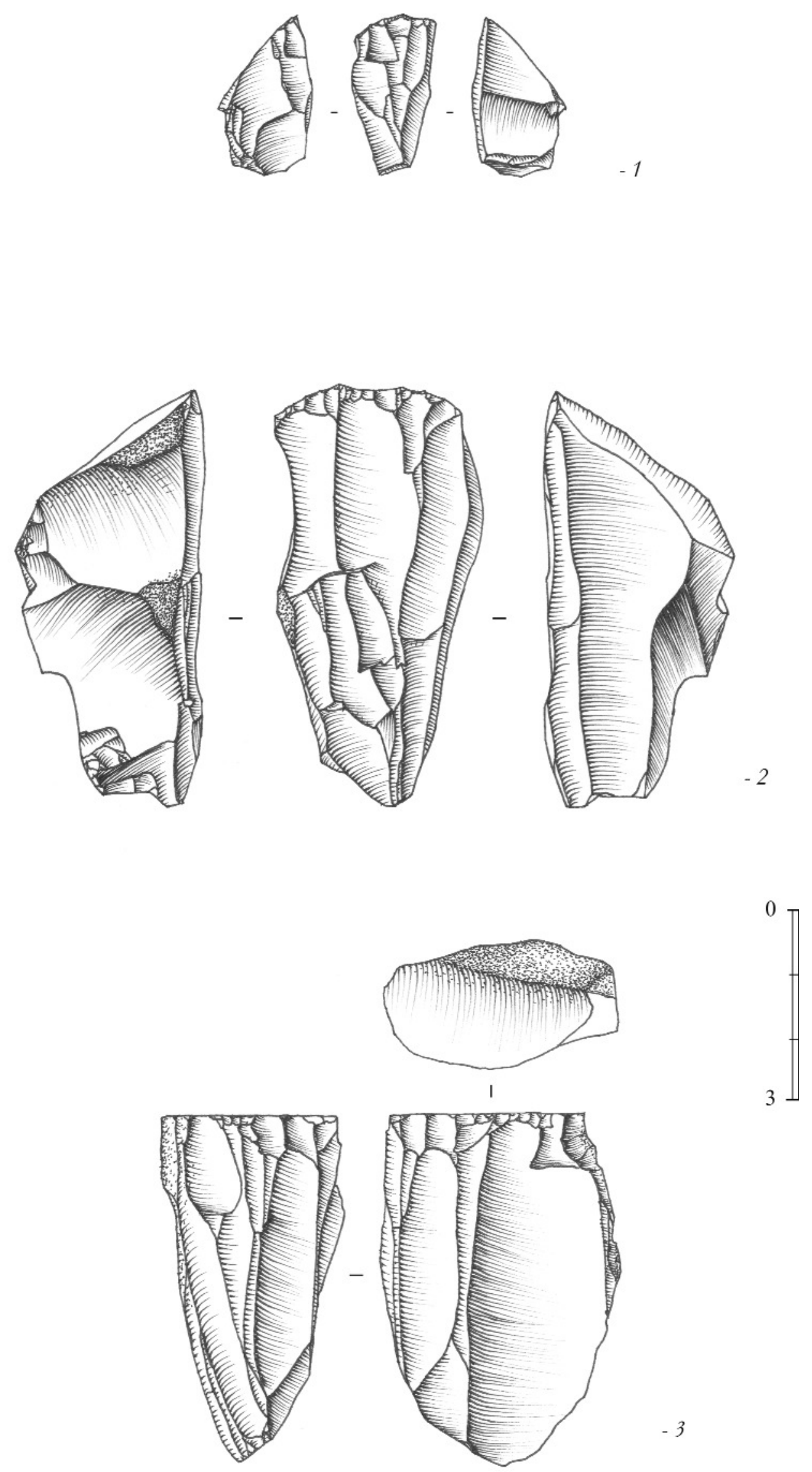

Figure 4 - Grotte de Font-Yves : Nucléus (dessins D. Pesesse).

Figure 4 - Grotte de Font-Yves : Cores (drawings D. Pesesse). 

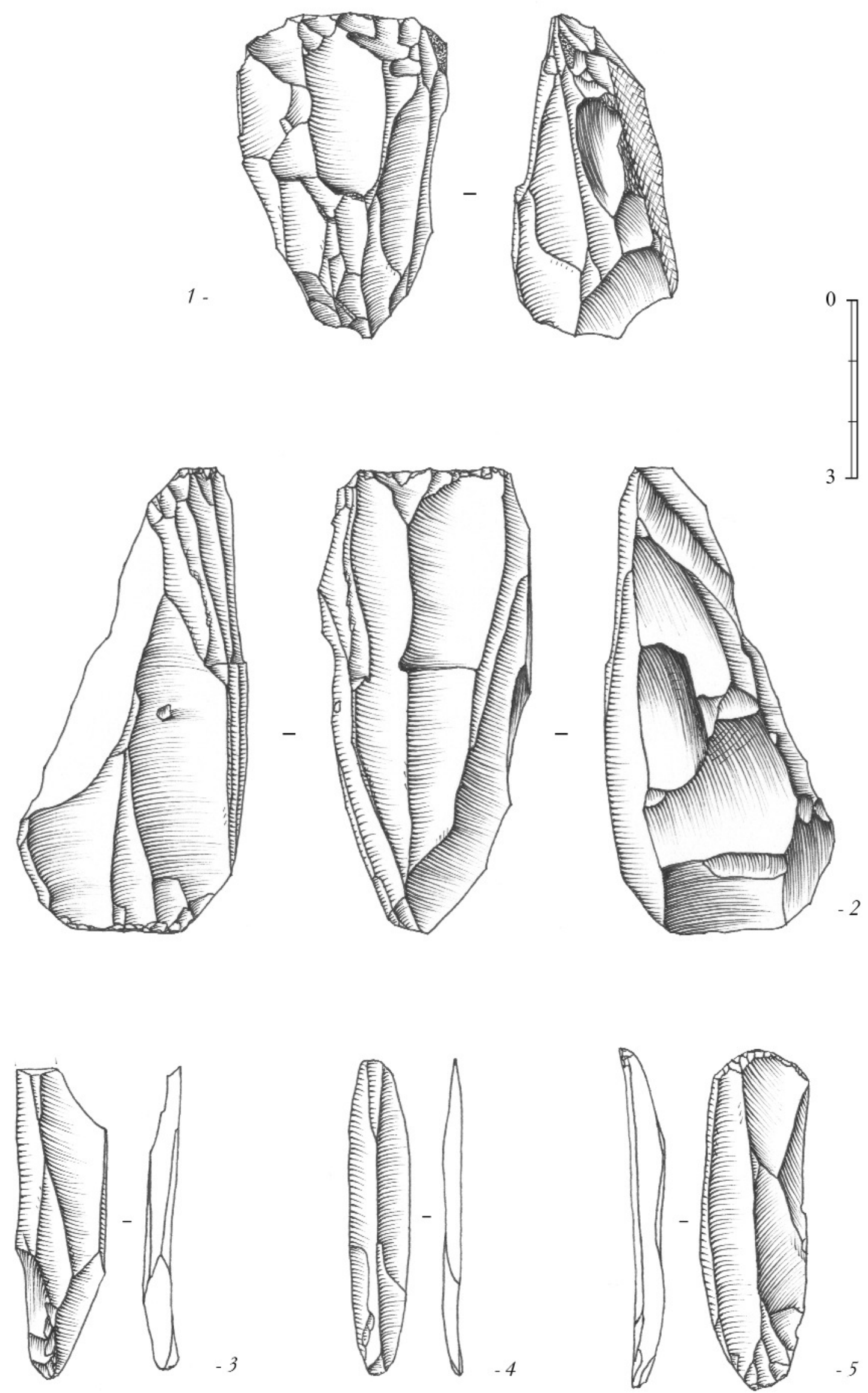

Figure 5 - Grotte de Font-Yves : Nucléus, produit et sous-produits (dessins D. Pesesse).

Figure 5 - Grotte de Font-Yves : Cores, product and by-products (drawings D. Pesesse). 

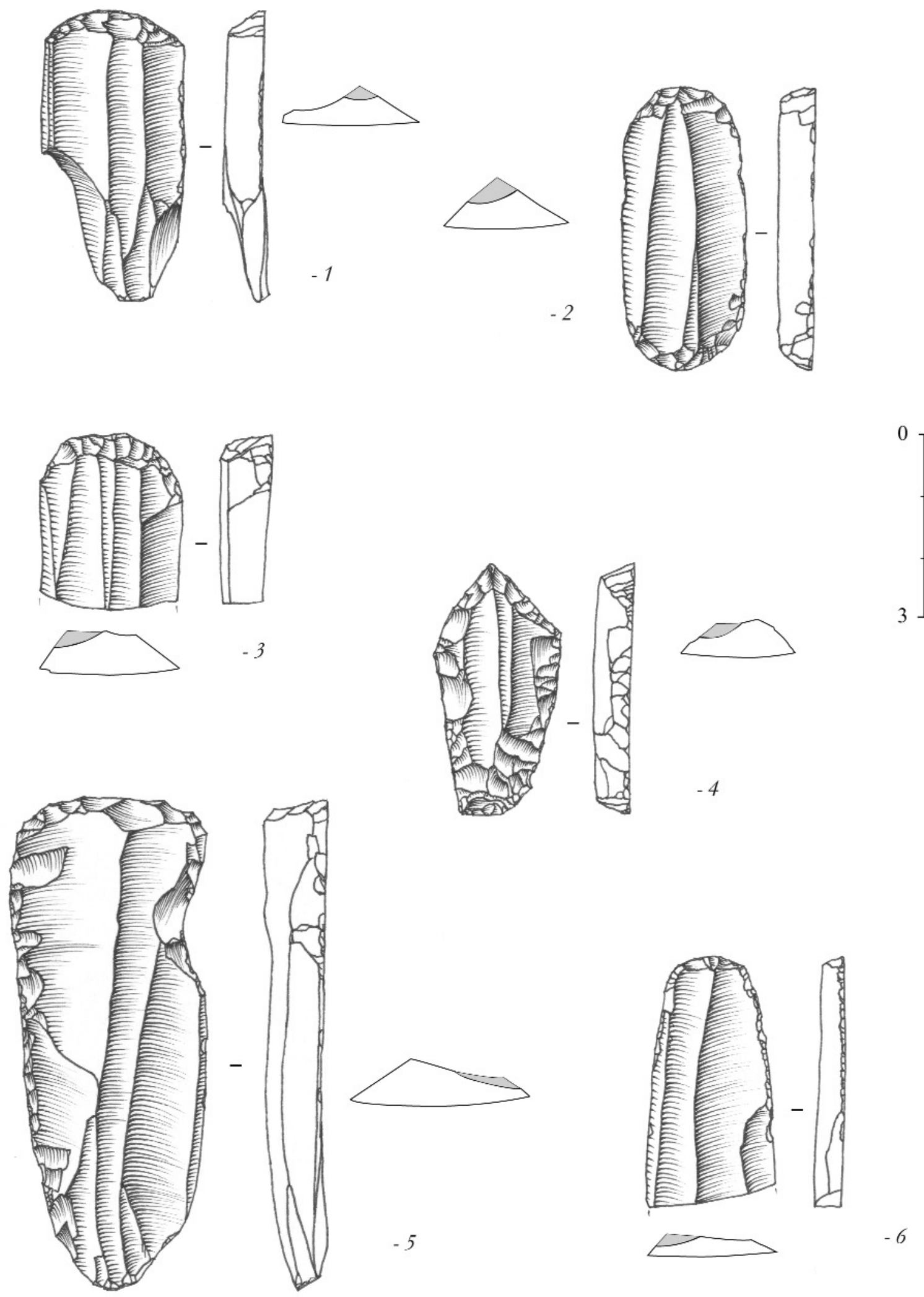

Figure 6 - Grotte de Font-Yves : Lames (dessins D. Pesesse).

Figure 6 - Grotte de Font-Yves : Blades (drawings D. Pesesse). 

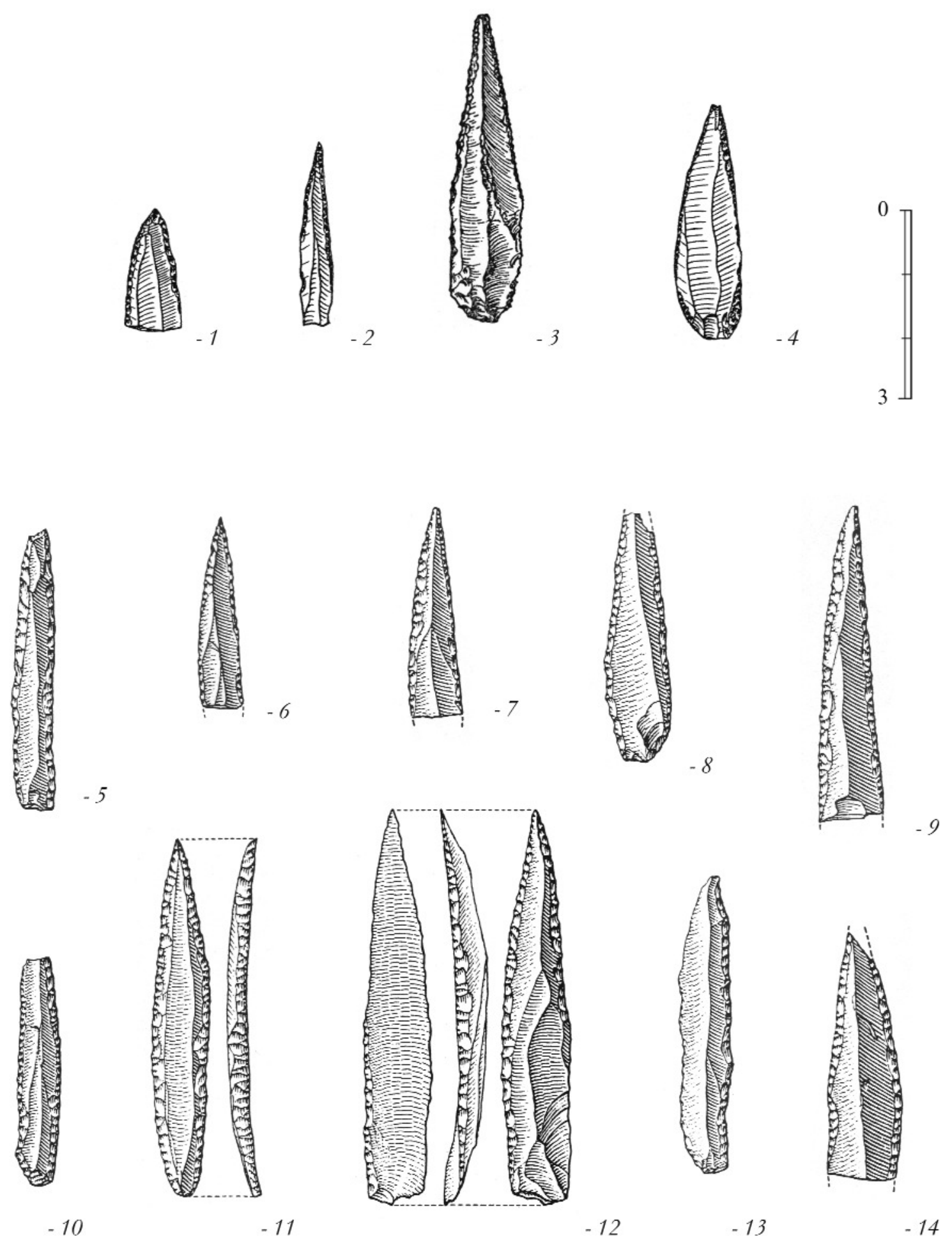

$-12$

$-13$

$-14$

Figure 7 - Pseudos pointes de Font-Yves, $n^{\circ} 1$ : Jabrud, c. 2 ; $n^{\circ} 2$ : Jabrud, c. $4 ; n^{\circ} 3$ : Jabrud ; $n^{\circ} 4:$ Predmosti ; $n^{\circ} 5$ à 14 :le Piage ( $n^{\circ}$ 1, 2, 4 : Sonneville-Bordes et Perrot et al. $1956 ; n^{\circ} 3$ : Heinzelin de Breaucourt $1962 ; n^{\circ} 5$ à 14 : Champagne et Éspitalié 1981).

Figure 7 - Pseudo Font-Yves points, $n^{\circ} 1$ : Jabrud, c. $2 ; n^{\circ} 2$ : Jabrud, c. $4 ; n^{\circ} 3:$ Jabrud, $n^{\circ} 4$ : Predmosti ; $n^{\circ} 5$ à 14 : le Piage $\left(n^{\circ} 1,2,4\right.$ : Sonneville-Bordes et Perrot et al. $1956 ; n^{\circ} 3$ : Heinzelin de Breaucourt $1962 ; n^{\circ} 5$ to 14 : Champagne et Éspitalié 1981). 
Cet amalgame avec les pièces de Krems-Hundssteig (Strobl, Obermaier 1909) revient à plusieurs reprises (Bohmers 1947 ; Schwabedissen 1954). J. Hahn, en revanche, distingue deux types au sein de l'outillage lamellaire de Krems-Hundssteig (Hahn 1977) :

- les pointes de Font-Yves qui présentent une retouche bilatérale directe ${ }^{4}$ et qui se différencient des pointes de FontYves éponymes par le caractère pointu (fig. 8, $\mathrm{n}^{\circ} 1$ à 5) ; - les pointes de Krems, limitées aux pièces à retouches alternes, se différenciant ainsi de la définition de H. Schwabedissen (fig. 8, $n^{\circ} 6$ à 9).

Mais cet auteur préconise l'abandon des deux termes et préfère celui de pointes à retouches marginales (Hahn 1977). Là encore, la définition de la pointe de Krems selon J. Hahn a par la suite été employée à tort pour désigner les pointes à retouches directes du site autrichien.

En parallèle, des pointes sont dénommées « de FontYves " dans l'Aurignacien du Levant et assimilées aux pièces d'El Wad (Garrod 1934, 1951, 1954, 1955). Ce rapprochement avec le Proche-Orient rentre bientôt dans la typologie française.
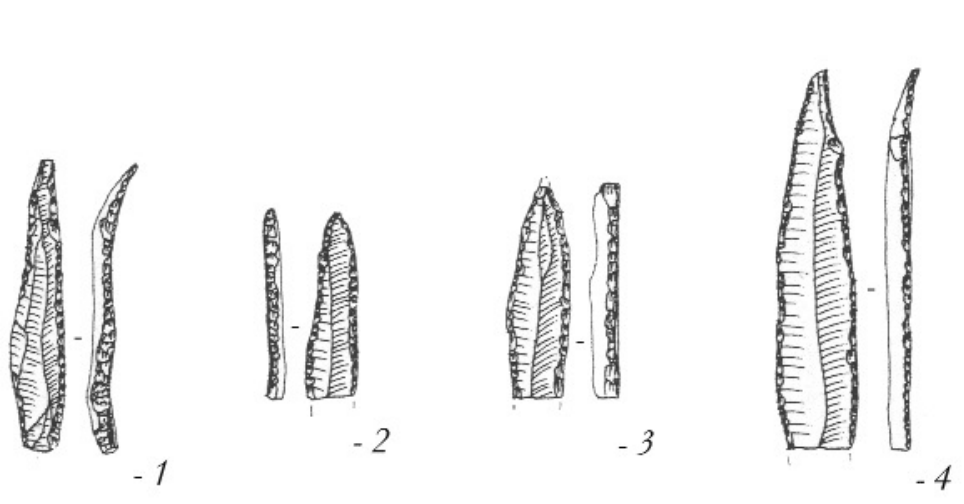

En 1956, D. de Sonneville-Bordes et J. Perrot donnent une définition très restreinte de l'armature (Sonneville-Bordes, Perrot 1956 - p. 547) : "Pointe à courtes retouches bilatérales semi-abruptes sur petite lame mince et étroite ou sur lamelle " et figurent deux pièces, provenant de Jabrud et de Predmosti (fig. 7, $n^{\circ} 1,2$, 4). Les exemplaires syriens, comme le fragment tchèque partagent peu de caractères avec les pointes de Font-Yves, si ce n'est la retouche directe et le support lamellaire. G. Laplace remarquait déjà cette incohérence (Laplace 1961 - p. 166). Deux ans plus tard, J. de Heinzelin retient aussi une définition trop limitée (Heinzelin de Breaucourt 1962). II figure un exemplaire de Jabrud (fig. $7, \mathrm{n}^{\circ} 3$ ) et reprend l'équation pointe de Font-Yves synonyme de pointe de Krems. M. Brézillon ne rétablit pas la situation en ajoutant que les pointes de Font-Yves ont la même diversité que les pointes de Krems (Brézillon 1968). Enfin, la corrélation FontYves/Krems fut reprise par P.-Y. Demars, qui précise par ailleurs que les pièces trouvées dans des industries aurignaciennes riches en lamelles Dufour diffèrent légèrement (Demars, Laurent 1989).

D'autres jalons sont vraisemblablement à rechercher pour illustrer ce glissement, initié avant même la première publi-
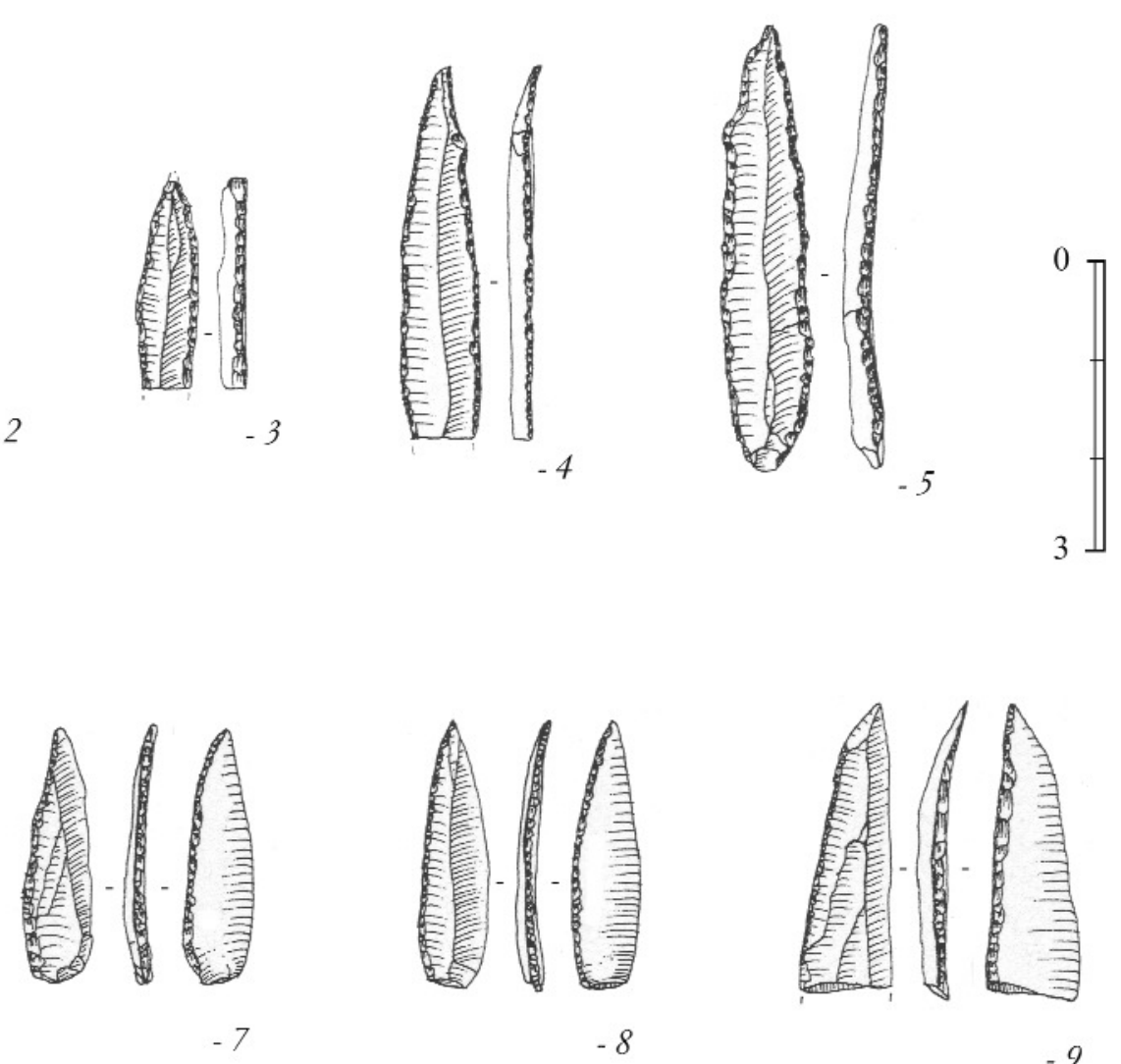

$-9$

Figure 8 - Krems-Hundssteig : $n^{\circ} 1$ à $5:$ : pointes de Font-Yves pointues » d'après J. Hahn $n^{\circ} 6$ à 9 : pointes de Krems (Hahn, 1977, dessins J. Hahn).

Figure 8 - Krems-Hundssteig : $n^{\circ} 1$ to $5:$ : pointed Font-Yves points » after J. Hahn, $n^{\circ} 6$ to 9 : Krems points (Hahn, 1977, drawings J. Hahn).

(4) Bien que le terme « direct » ne soit pas précisé, il est implicite au regard des illustrations et de la définition du deuxième groupe. 
cation de Font-Yves. Toujours est-il qu'en un temps relativement court, la pointe corrézienne rencontra une pièce considérée alors comme analogue en Autriche, la pointe de Krems, atteignit les rivages du Levant et réintégra la typologie française complètement dénaturée.

Se fondant sur ces définitions très incomplètes, voire erronées, ces «nouvelles pointes de Font-Yves » furent par la suite retrouvées au Piage (Champagne, Espitalié 1981), à l'Arbreda (Maroto et al. 1996) et sur d'autres gisements. Actuellement, les pointes de Font-Yves désignent les pointes axiales du premier Aurignacien (citons par exemple Bon 2000, 2002 ; Bordes 2002, 2005 ; Ortega et al. 2005 ; Normand 2002 ; Normand, Turq 2005 ; Normand et al. 2007 ; Tsanova 2008).

Une suite de glissements eut lieu, d'abord liés à la vague convergence morphologique des spécimens autrichiens, tchèques et syriens. La convergence fut considérée ensuite comme élément premier de définition, recouvrant alors celle de la pointe de Krems sous couvert de variabilité morphologique. L'absence de nouvelles séries importantes de pointes de Font-Yves a certainement contribué à maintenir la confusion. Ces pointes ont été intégrées à la variabilité inter-site des pointes à retouches bilatérales du premier Aurignacien dans un espace géographique large.

Les pointes éponymes ne peuvent donc être rapportées à une occupation du Protoaurignacien. A ce titre, l'absence de grandes lamelles Dufour à Font-Yves est aussi significative que les différences évoquées.

Maintenir cette appellation pour les pièces appointées du Protoaurignacien entretient donc la confusion. Reprendre la proposition de G. Laplace et distinguer les pointes de Font-Yves des lamelles de Font-Yves ne conduirait qu'à prolonger cet amalgame. Ce terme ne devrait donc plus être appliqué aux séries des débuts de l'Aurignacien.

\section{Quelle attribution culturelle pour les pointes de Font-Yves?}

Ayant fouillé la Font-Robert avant Font-Yves, c'est dans une publication ultérieure, que les abbés reconnurent ces pointes dans le premier abri (Bouyssonie 1923). La reprise des collections permit à P.-Y. Demars et G. Mazière d'individualiser deux de ces pointes (Mazière 1978 ; Demars 1994). Plusieurs spécimens sont en effet présents (fig. 9). Localement, G. Mazière a également reconnu trois exemplaires à la grotte du Loup (Mazière 1978). Dans ce secteur, elles sont associées à de nombreuses lamelles à retouches alternes que l'auteur ne qualifie pas de lamelle Dufour car, par rapport aux lamelles retouchées de la Grotte Dufour, elles "sont réalisées sur un support différent (lamelles torses, étroites, nettement plus allongées) 》 (Mazière 1978 - p. 150).

A Chanlat, un spécimen entier, issu du niveau supérieur, présente des bords parallèles et des extrémités peu aiguës
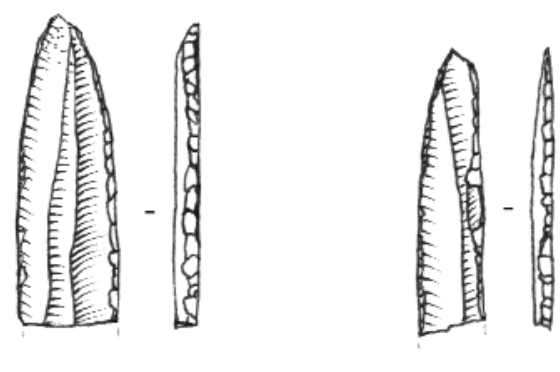

0

3

$-1$

$-2$

Figure 9 - Grotte de la Font-Robert : probables pointes de Font-Yves (dessins D. Pesesse).

Figure 9 - Grotte de la Font-Robert : probable Font-Yves points (drawings $D$. Pesesse).

caractéristiques (Bouyssonie, Delsol 1950). J. Bouyssonie décrit ainsi la collection, par rapport au niveau sousjacent : "Les burins sont en plus forte proportion, surtout ceux que j'ai appelés burins busqués avec encoche, à propos du Bouitou supérieur et burins carénés plans " (i.e. burins des Vachons) (Bouyssonie, Delsol 1950 - p. 188). Les fines lamelles retouchées disparaissent pour « une jolie lame mince, et à bords presque parallèles, à fines retouches presque abruptes, du type Font-Yves " (Bouyssonie, Delsol 1950 - p. 188). Tout en questionnant sa réelle homogénéité, le niveau supérieur de Chanlat pourrait globalement être assimilé à un Aurignacien récent.

Ces trois sites corréziens ne clarifient pas catégoriquement la position chrono-stratigraphique des pointes de FontYves, mais livrent certaines données relatives à leur position stratigraphique, leur association ou leur exclusion avec d'autres marqueurs culturels bien représentés régionalement. A Chanlat, gisement dont la stratigraphie a été au moins partiellement reconnue, cette pointe est présente dans un niveau sus-jacent à un ensemble comprenant des grandes lamelles Dufour. De même, dans les séries à grandes lamelles à retouches alternes de Dufour (Bouyssonie 1944) et Bos-del-Ser (Bouyssonie 1923), aucune pointe de Font-Yves n'est présente. Au Loup, ces pointes sont associées à des lamelles à retouches alternes de profil tors, caractéristiques qui pourraient éventuellement évoquer une phase récente de l'Aurignacien. Cependant, l'absence de documentation publiée sur la stratigraphie des occupations aurignaciennes et le matériel afférent limitent l'interprétation de cette donnée.

En Périgord, à l'Abri Pataud, plusieurs fragments de lamelles retouchées ont été attribués par H.L. Movius au type Font-Yves. Si ces artefacts sont effectivement des pointes de Font-Yves, cela permettrait de les replacer dans un cadre chrono-stratigraphique bien défini. Vérifions les arguments en faveur de cette appellation.

\section{L’Abri Pataud}

L'importante séquence de l'Abri Pataud constitue un des meilleurs observatoires de l'évolution des systèmes lithiques compte tenu du nombre, de la qualité des niveaux 
et de la documentation pour l'Aurignacien et le Gravettien (Bricker 1995 ; Chiotti 1999 ; Agsous et al. 2006). Le dernier niveau aurignacien, la couche 6 , est situé en stratigraphie entre un niveau à burins busqués, la couche 7 , et un niveau dominé par les fléchettes et les armatures à dos, la couche 5. Au niveau régional, sa position chrono-stratigraphique s'avère extrêmement originale. La couche 6 peut être perçue comme l'un des derniers enregistrements archéologiques des traditions " aurignaciennes ". Deux datations à $29347 \pm 1374 \mathrm{BP}$ et $28510 \pm 280 \mathrm{BP}(\mathrm{GrN} 6273)$ sont en accord avec le caractère récent de cette occupation (Bricker 1995).

Au regard des niveaux antérieurs, certains choix économiques spécifiques mais aussi une certaine diversité des chaînes opératoires ont pu être mis en évidence (Chiotti 1999, 2003). En effet, cette industrie reflète des évolutions majeures des normes aurignaciennes par l'apparition d'objectifs originaux et de modalités de production inédites. Cela se traduit par une très forte diversification des produits dont la recherche de petites lames et de lamelles rectilignes, pointues et très régulières (Pesesse 2008a, 2010). La faiblesse de la fraction retouchée, en raison de l'absence de tamisage lors de la fouille en 1961, restreint la compréhension de l'ensemble des objectifs de la production, en particulier pour les gabarits les plus modestes. Toutefois, un minimum de huit pièces retouchées se démarque. Ces outils ont été attribués au type Font-Yves par H.L. Movius, appellation reprise par la suite par A.S. Brooks et L. Chiotti (fig. 10, $\mathrm{n}^{\circ} 1$ à 6 ).

Les supports utilisés sont de petites lames ou de grandes lamelles. En dépit de leur fragmentation, une nette tendance à la rectitude est observable. Ces pièces sont retouchées sur un bord, de préférence le droit, ou les deux par une retouche d'intensité variable. Les bords de ces lamelles sont parallèles. Les deux bases sont de morphologie ogivale (fig. $10, \mathrm{n}^{\text {os }} 4$ et 6 ).

En dépit de la fragmentation et de la faiblesse de ce corpus, ces lamelles retouchées possèdent plusieurs points communs avec les pointes de Font-Yves du gisement du Bassin de Brive. Elles présentent un rapport largeur/épaisseur proche des pièces éponymes. La morphologie en segment de cercle, définie par J. Bouyssonie, se retrouve également. L'intensité de la retouche, de marginale à semiabrupte, varie de même. Les deux bases de pointes de Pataud reprennent la morphologie ogivale des pièces éponymes. A cela, s'ajoute un dernier critère diagnostique, commun à ces deux gisements : une pièce de Pataud (fig. $11, \mathrm{n}^{\circ} 2$ ) révèle l'orientation oblique à gauche des retouches du bord droit, élément tributaire d'un procédé de façonnage identique et original (fig. $11, n^{\circ} 1$ et 3 ).

A Pataud, l'origine des supports de ces pointes peut être recherchée parmi une production réalisée sur blocs notamment. Un nucléus correspond à cet objectif ; il présente une structure unipolaire convergente dont les flancs sont définis par des enlèvements extraits depuis la base du volume (fig. 10, $\mathrm{n}^{\circ} 7$ ). Ce schéma témoigne également de fortes affinités avec le matériel de Font-Yves (fig. 5 et 6 ).

D'autres points communs existent entre ces assemblages, notamment la présence d'un mode de production lamellaire original à partir d'un pan de la face supérieure de l'éclat-nucléus ${ }^{5}$, de débitages réalisés à partir de burins des Vachons ou de burins mixtes busqués-vachons.

Sur ces bases, les fragments de lamelles retouchées de l'Abri Pataud peuvent être effectivement rapprochés du type Font-Yves. Le partage de conventions dans le mode de production et de façonnage, notamment dans certains aspects discrets, confirme l'attribution de H.L. Movius. Auteur d'une synthèse sur les gisements corréziens (Movius 1980), H.L. Movius connaissait probablement le matériel du gisement de Font-Yves, ce qui expliquerait la justesse de l'attribution.

\section{La Grotte Bouyssonie}

Un siècle après la fouille de la Grotte de Font-Yves, T. Bismuth a découvert en mars 2005 un gisement sur le versant nord du plateau de Bassaler à quelques dizaines de mètres de la Grotte de Bassaler (Couchard, SonnevilleBordes 1960). En mars 2006, L. Detrain entreprit une opération de diagnostic mettant au jour une importante stratigraphie couvrant l'essentiel du Paléolithique supérieur, de l'Aurignacien au Magdalénien, dont un niveau à pointes de Font-Yves (Detrain et al. 2006 ; Detrain 2006). Sur ce site, la Grotte Bouyssonie (Brive-la-Gaillarde, Corrèze), une fouille programmée a débuté en août 2008, confirmant l'importance de ce gisement et notamment du niveau à pointes de Font-Yves (Pesesse 2008b). Pour l'heure, plus d'une quarantaine de ces pointes a été trouvée sur une surface d'1 $\mathrm{m}^{2}$.

Ces pièces partagent l'ensemble des caractéristiques des pièces éponymes : standardisation, latéralisation, mode de façonnage (fig. 12). La morphologie des extrémités, l'inclinaison de la retouche sur certains spécimens sont identiques aux pièces éponymes. La seule particularité concerne la répartition des gabarits. Avec des pointes de dimensions identiques aux pièces originales, un module extrêmement réduit est présent. Compte tenu des méthodes de fouille du tout début du XXe siècle, ces outils nanolithiques (la plus petite pointe entière mesure $9 \mathrm{~mm}$ de longueur) sont absents de la série éponyme. En revanche, plusieurs nucléus de la Grotte de Font-Yves recoupent cette gamme de gabarit et présentent des enlèvements de morphologie compatible.

Des réponses pourront donc être apportées sur l'âge exact et les caractéristiques de cette industrie restée " orpheline » un siècle durant. 

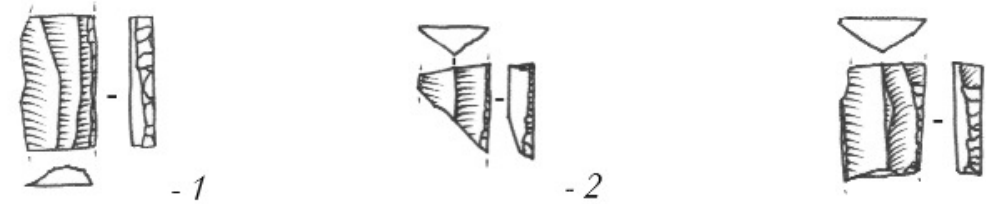

$-3$
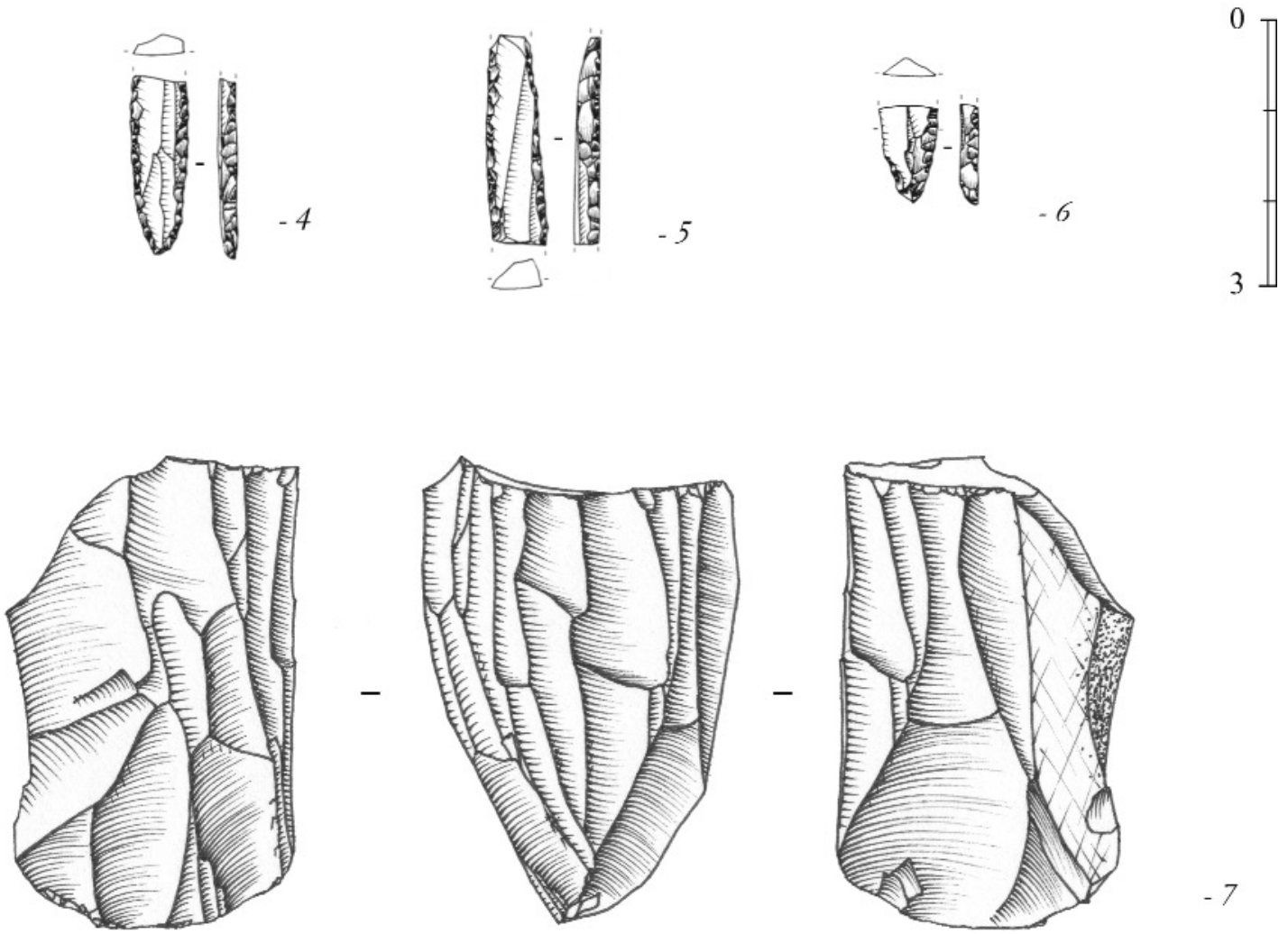

Figure 10 - Abri Pataud, couche $6: n^{\circ} 1$ à 6 : pointes de Font-Yves, $n^{\circ} 7:$ nucléus (dessins $n^{\circ} 1$ à $3, n^{\circ} 7$ : D. Pesesse ; $n^{\circ} 4$ à 6 : L. Chiotti).

Figure 10 - Abri Pataud, layer $6: n^{\circ} 1$ à 6 : Font-Yves points, $n^{\circ} 7:$ core (drawings $n^{\circ} 1$ to $3, n^{\circ} 7: D$. Pesesse ; $n^{\circ} 4$ to 6 : L. Chiotti).

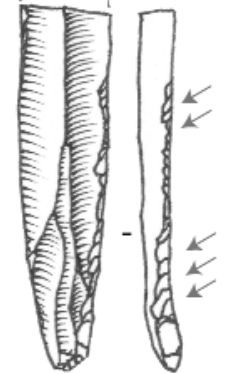

$-1$

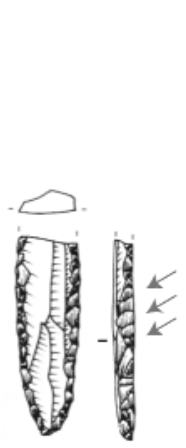

$-2$
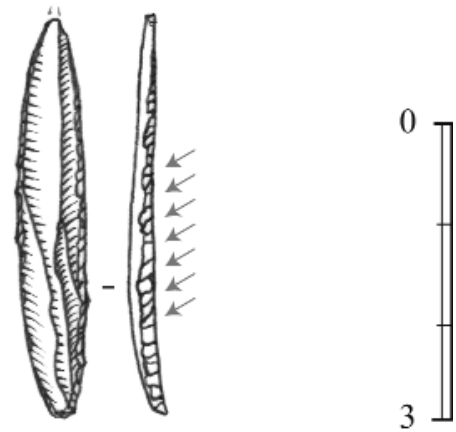

$-3$

Figure 11 - Inclinaison de la retouche des pointes de Font-Yves, $n^{\circ} 1$ : Grotte de Font-Yves, $n^{\circ} 2$ : Abri Pataud, couche 6, $n^{\circ} 3$ : Grotte Bouyssonie (dessins $n^{\circ} 1$ et $3: D$. Pesesse ; $n^{\circ} 2$ : L. Chiotti).

Figure 11 - Angle retouch of the Font-Yves points, $n^{\circ} 1$ : Grotte de Font-Yves, $n^{\circ} 2$ : Abri Pataud, layer 6, $n^{\circ} 3$ : Grotte Bouyssonie (drawings $n^{\circ} 1$ and $3: D$. Pesesse ; $n^{\circ} 2$ : L. Chiotti). 


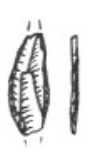

$-1$

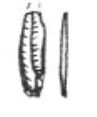

$-2$<smiles>[LiH]</smiles>

$-3$

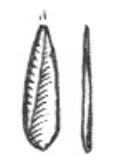

$-4$

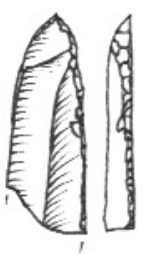

$-6$

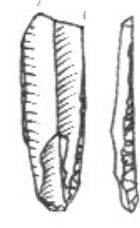

$-7$

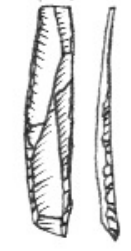

$-8$
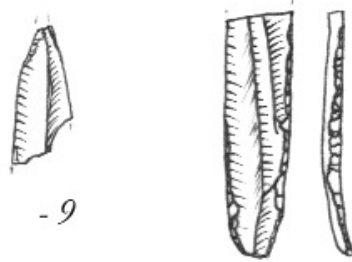

$-10$
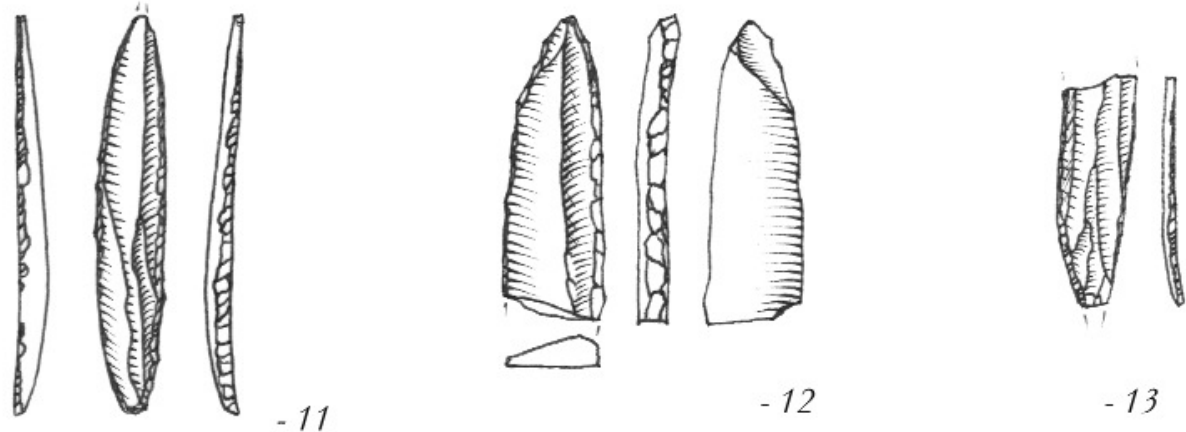

$-13$

Figure 12 - Grotte Bouyssonie : pointes de Font-Yves (dessins D. Pesesse).

Figure 12 - Grotte Bouyssonie : Font-Yves points (drawings D. Pesesse).

\section{Conclusion}

Le terme " pointe de Font-Yves » a connu un large succès aux dépens des caractéristiques spécifiques aux pointes éponymes. L'ancienneté des fouilles de la Grotte de FontYves et l'absence d'assemblages comparables ont probablement contribué à cette perte de sens. Mais le caractère succinct et partial des définitions établies par les auteurs successifs a tout d'abord généré puis accentué ce glissement terminologique.

A ces problèmes de définition répond l'absence de questionnement quant à la cohérence de l'industrie de la Grotte de Font-Yves, composée d'un fonds classique et d'un outillage lamellaire original. A ce titre, la prudence des premiers auteurs, les abbés J. et A. Bouyssonie et L. Bardon, mérite d'être soulignée.

Compte tenu des différences avec les pointes à retouches directes du Protoaurignacien, le terme « pointe de FontYves " doit désormais être limité dans son usage aux exemplaires correspondant aux pièces éponymes. La présence d'une base ogivale, d'une retouche inclinée, d'un apex peu aigu, la très faible variation morphologique interindividuelle et un mode de production soigné comptent parmi les caractéristiques princeps de définition. D'autres appellations sont donc à rechercher pour désigner les pointes du Protoaurignacien, un terme neutre «pointe à retouches directes », « lamelle appointée » ou bien le nom d'un site par exemple. De plus, dans le contexte du Protoaurignacien, ces pointes méritent d'être caractérisées quant à leurs spécificités, leur réelle homologie inter-site et leur place face aux différents groupes - pointes de Krems, d'El Wad, d'Arjeneh.

Jusque-là considérée comme une armature lithique développée par les premiers Aurignaciens, la pointe de Font-Yves traduit plus vraisemblablement l'une des dernières innovations aurignaciennes dans le domaine des industries lithiques. En effet, les pièces de la couche 6 de l'Abri Pataud constituent à l'heure actuelle un meilleur point de comparaison. La position de ces pointes en sommet de 
séquence aurignacienne pourra être vérifiée dans le cadre de la fouille de la Grotte Bouyssonie dans un avenir proche.

Cette réattribution modifie profondément notre compréhension de la fin de l'Aurignacien et des processus historiques à l'origine de la structuration du Gravettien. Jusque-là, les savoir-faire connus pour les groupes de l'Aurignacien récent en matière d'équipement lithique différaient des productions documentées pour le début du Gravettien. En effet, les productions lamellaires réalisées à partir de burins busqués, des micro-lamelles torses (Lucas 1997, 2000 ; Chiotti 1999, 2003 ; Bordes 2005), répondent à des objectifs très éloignés des armatures axiales rectilignes connues pour le premier Gravettien (Pesesse 2008a, 2008c, 2008d). La mise en évidence récente d'un concept de production lamellaire à partir du « burin des Vachons » a permis d'entrevoir certaines modifications importantes dans les objectifs de production chez les populations de l'Aurignacien récent (Pesesse, Michel 2006). Toutefois, le manque de données précises concernant les objectifs de cette production ne permet pas encore d'apprécier pleinement la place de ce schéma opératoire dans les processus de restructuration des systèmes techniques à la fin de l'Aurignacien. L'industrie de la couche 6 de l'Abri Pataud apporte un éclairage déterminant quant à cette question (Pesesse 2010). Elle permet d'envisager une évolution profonde des objectifs à la toute fin de cette période, orientés vers la recherche d'armatures axiales rectilignes en silex. La pointe de Font-Yves peut ainsi être considérée comme l'un des principaux témoins des évolutions des systèmes techniques à la fin de l'Aurignacien. Ce type de production, classiquement rapportée au Gravettien, trouve ainsi des prémisses aurignaciennes. De fait, la structuration du Gravettien trouve un ancrage régional au sein des systèmes techniques développés par les derniers aurignaciens.

\section{Remerciements}

Mes remerciements s'adressent à Thierry Bismuth, Roland Nespoulet et Laurent Chiotti.

\section{Références bibliographiques}

AGSOUS S., LENOBLE A., NESPOULET R., 2006 - L'Abri Pataud. In Texier J.P. (dir.) Livret-guide de l'excursion de la Société géologique française "Sites préhistoriques du Périgord », Pôle international de la Préhistoire.

BARDON L., BOUYSSONIE A., BOUYSSONIE J. 1920 - La station préhistorique de Font-Yves (Corrèze), Bulletin de la Société Scientifique, Historique et Archéologique de la Corrèze, t. 42, p. 291-301

BOHMERS A. 1947 - Jong-Palaeolithicum en VroegMesolithicum. Gedenkboek A.E. van Giffel, Meppel.
BON F. 2000 - La question de l'unité technique et économique de l'Aurignacien : réflexions sur la variabilité des industries lithiques à partir de l'étude comparée de trois sites des Pyrénées françaises (la Tuto de Camalhot, Régismont-le-Haut et Brassempouy), Mémoire de Doctorat, Université de Paris I.

BON F. 2002 - L'Aurignacien entre mer et océan : réflexion sur l'unité des phases anciennes de l'Aurignacien dans le sud de la France. Mémoire de la Société Préhistorique Française, vol. 29.

BORDES J.-G. 2002 - Les interstratifications Châtelperronien/Aurignacien du Roc-de-Combe et du Piage (Lot, France). Analyse taphonomique des industries lithiques ; conséquences archéologiques. Mémoire de Doctorat. Université de Bordeaux I.

BORDES J.-G. 2005 - La séquence aurignacienne du Nord de l'Aquitaine : variabilité des productions lamellaires à Caminade-Est, Roc-de-Combe, le Piage et CorbiacVignoble II, In : Le Brun-Ricalens F. (Ed.), Productions lamellaires attribuées à l'Aurignacien: Chaînes opératoires et perspectives techno-culturelles. XIV congrès de I'UISPP, Liège (2-8 septembre 2001). Luxembourg : ArchéoLogiques n 1, p. 123-154.

BOUYSSONIE J. 1923 - Station préhistorique de Bos-delSer près Brive (Corrèze), Association Française pour l'Avancement des Sciences, $47^{ }$session, Bordeaux, p. 617-622.

BOUYSSONIE J. 1944 - La grotte Dufour près Brive (Corrèze), Bulletin de la Société Préhistorique Française, t. 43-44, p. 186-192.

BOUYSSONIE A., BOUYSSONIE J., BARDON L. 1913 - La station préhistorique de Font-Yves (Corrèze), Revue Anthropologique, t. 23, p. 218-225.

BOUYSSONIE J., DELSOL H. 1950 - La Grotte de Chanlat, près Brive (Corrèze), Congrès Préhistorique de France, XIII ${ }^{\mathrm{e}}$ session, Paris, p. 183-190.

BREUIL H. 1912 - Les subdivisions du Paléolithique supérieur et leur signification, Congrès International d'Anthropologie et d'Archéologie Préhistoriques, XIVe session, Genève.

BREUIL H. 1937 - Les subdivisions du Paléolithique supérieur et leur signification, Congrès International d'Anthropologie et d'Archéologie Préhistoriques, XIV session, $2^{\mathrm{e}}$ édition, Genève.

BREZILLON M. 1968 - La dénomination des objets de pierre taillée. Matériaux pour un vocabulaire des préhistoriens de langue française, IV suppl. à Gallia Préhistoire, $417 \mathrm{p}$.

BRICKER H. M. (dir.) 1995 - Le Paléolithique supérieur de l'Abri Pataud (Dordogne) : les fouilles de H.L. Movius, Paris, Maison des Sciences de l'Homme, Documents d'Archéologie Française, $n^{\circ}$ 50, 328 p. 
CHAMPAGNE F., ESPITALIE R. 1981 - Le Piage, site préhistorique du Lot. Mémoires de la Société Préhistorique Française, 15, $205 \mathrm{p}$.

CHIOTTI L. 1999 - Les industries lithiques de l'Aurignacien de l'Abri Pataud, les Eyzies-de-Tayac (Dordogne) : étude technologique et typologique, Mémoire de Doctorat, Museum d'Histoire Naturelle, Institut de Paléontologie Humaine, Paris.

CHIOTTI L. 2003 - Les productions lamellaires dans l'Aurignacien de l'Abri Pataud, Les Eyzies-de-Tayac (Dordogne), Gallia Préhistoire, t. 45, p. 113-156.

COUCHARD J., SONNEVILLE-BORDES D. (de) 1960 - La grotte de Bassaler-Nord, près de Brive, et la question du Périgordien II en Corrèze, L'Anthropologie, t. 64, p. 415437.

DEMARS P.-Y. 1992 - L'Aurignacien ancien en Périgord. Le problème du Protoaurignacien, Paléo, t. 4, p. 101-117.

DEMARS P.-Y. 1994 - L'économie du silex au Paléolithique supérieur dans le Nord de l'Aquitaine, Thèse d'Etat, Université de Bordeaux I, 2 vol

DEMARS P.-Y., LAURENT P. 1989 - Types d'outils lithiques du Paléolithique supérieur en Europe, Cahiers du Quaternaire, 14, Paris, Presses du CNRS.

DETRAIN L. 2006 - Rue Roger Nayrac, Brive-la-Gaillarde, Bilan Scientifique, Limousin, p. 12-13.

DETRAIN L. avec la collaboration de Bertran P., Chevreuse F., Colonge D., Boulogne S., O'YI W. 2006 - Rue Roger Nayrac, Brive, Rapport de diagnostic, $45 \mathrm{p}$.

GARROD D.A.E. 1934 - The Stone Age of Palestine, Antiquity, vol VIII, p. 133-150.

GARROD D.A.E. 1951 - A transitional industry from the base of the upper paleolithic in Palestine and Syria, Journal of the Royal Anthropological Institut of Great Britain and Ireland, vol. $81, \mathrm{n}^{\circ} 1 / 2$, p. 121-130.

GARROD D.A.E. 1954 - Excavations at the Mugharet Kebara, Mount Carmel, 1931 : The Aurignacian industries, Proceedings of the Prehistoric Society, vol. XX, pt, 2, p. $155-192$.

GARROD D.A.E. 1955 - The Mugharet el-Emireh in lower Galilee : Type-station of the Emiran Industry, Journal of the Royal Anthropological Institute of Great Britain and Ireland, vol. 85, pt. I-II.

HAHN J. 1977 - Aurignacien. Das ältere Jungpaläolithikum in Mittel- und Osteuropa. Fundamenta A/9, Köln, Wien.

HEINZELIN DE BREAUCOURT J. (de) 1962 - Manuel de typologie des industries lithiques, Bruxelles, $74 \mathrm{p}$.

LALANNE J.G., BOUYSSONIE J. 1941 à 1946 - Le gisement paléolithique de Laussel. Fouilles du Docteur Lalanne, L'Anthropologie, t. 50, p. 1-163.
LAPLACE G. 1958-1961 - Recherches sur l'origine et l'évolution des complexes leptolithiques. Le problème des Périgordiens I et II et l'hypothèse du Synthétotype aurignaco-gravettien. - Essai de typologie analytique, Quaternaria, vol. V, p. 153-240

LAPLACE G. 1966 - Recherche sur l'origine et l'évolution des complexes leptolithiques. Ecole Française de Rome, Mélanges d'Archéologie et d'Histoire, suppl. 4.

LUCAS G. 1997 - Les lamelles Dufour du Flageolet I (Bézenac, Dordogne) dans le contexte aurignacien, Paléo, $n^{\circ}$ 9, p. 191-219.

LUCAS G. 2000 - Les industries lithiques du Flageolet I (Dordogne) : approche économique, technologique, fonctionnelle et analyse spatiale, Mémoire de Doctorat, Université de Bordeaux I, 2 vol., 603 p.

MAROTO J., SOLER N., FULLOLA J.M. 1996 - Cultural change between middle and upper palaeolithic in Catalonia, in The last Neandertals, the first anatomically modern humans : a tale about the human diversity : cultural change and human evolution : the crisis at $40 \mathrm{Ka} \mathrm{BP}, \mathrm{E}$. Carbonell, M. Vaquero éds, Barcelona, Universitat Rovira i Virgili, p. 219-250.

MAZIERE G. 1978 - Le Paléolithique en Corrèze, Mémoire de Doctorat, Université de Paris $\mathrm{X}$.

MAZIERE G. 1980 - Circonscription du Limousin, Gallia Préhistoire, t. 23, fasc. 2.

MAZIERE G., TIXIER J. 1976 - Les civilisations du Paléolithique supérieur en Limousin, La Préhistoire française, t. 1, Les civilisations paléolithiques et mésolithiques de la France, IX ${ }^{\circ}$ Congrès UISPP, s.I.d. H. de Lumley, Nice, éds CNRS, p. 1281-1296.

MOVIUS H.L. 1980 - Les sites Aurignaco-périgordiens (Paléolithique supérieur) de Corrèze, Bulletin de la Société Scientifique, Historique et Archéologique de la Corrèze, t. 102.

NORMAND C. 2002 - L'Aurignacien de la Salle de SaintMartin (Grotte d'Isturitz, commune de Saint-Martin d'Arberoue, Pyrénées-Atlantiques) : données préliminaires sur l'industrie lithique recueillie lors des campagnes 20002002, Espacio, Tiempo y Forma, Série I, Prehistoria y Arqueologia, t. 15, p. 145-174.

NORMAND C., BEAUNE de S.-A., COSTAMAGNO S., DIOT M.-F., HENRY-GAMBIER D., GOUTAS N., LAROULANDIE V., LENOBLE A., O'FARRELL M., RENDU W., RIOS GARAIZAR J., SCHWAB C., TARRINO VINAGRE A., TEXIER J.-P., WHITE R. 2007 - Nouvelles données sur la séquence aurignacienne d'Isturitz (communes d'Isturitz et de Saint-Martin-d'Arberoue ; Pyrénées-Atlantiques), In : Un siècle de construction du discours scientifique en Préhistoire, vol. III "...Aux conceptions d'aujourd'hui », J. Evin éd., XXV| Congrès Préhistorique de France, Congrès du centenaire de la Société Préhistorique Française, Avignon, 21-25 septembre 2004, p. 277-293. 
NORMAND C., TURQ A. 2005 - L'Aurignacien de la Grotte d'Isturitz (France) : la production lamellaire dans la séquence de la salle de Saint-Martin, Productions lamellaires attribuées à l'Aurignacien : Chaînes opératoires et perspectives technoculturelles, In : Le Brun-Ricalens F. (Ed.), Productions lamellaires attribuées à l'Aurignacien : Chaînes opératoires et perspectives techno-culturelles. XIV congrès de I'UISPP, Liège 2-8 Septembre 2001, Archéologiques 1, Luxembourg, 2005, p. 375-392.

O'FARRELL M. 2004 - Les pointes de la Gravette de Corbiac (Dordogne) et considérations sur les armes de chasse au Paléolithique supérieur ancien, in Approches fonctionnelles en Préhistoire, s.l.d. P. Bodu, C. Constantin, 24-26 novembre 2000, Nanterre, XXV Congrès Préhistorique de France, Société Préhistorique Française, p. 121-138.

ORTEGA COBOS D., SOLER MASFERRER N., MAROTO GENOVER J. 2005 - La production des lamelles pendant l'aurignacien archaïque dans la grotte de l'arbreda : organisation de la production, variabilité des méthodes et des objectifs, In : Le Brun-Ricalens F. (Ed.), Productions lamellaires attribuées à l'Aurignacien: Chaînes opératoires et perspectives techno-culturelles. XIV ${ }^{\mathrm{e}}$ congrès de I'UISPP, Liège 2-8 Septembre 2001, ArchéoLogiques 1, Luxembourg, 2005, p. 359-373.

OTTE M., BIGLARI M., FLAS D., SHIDRANG S., ZWYNS N., MASHKOUR M., NADERI R., MOHASEB A., HASHEMI N., DARVISH J., RADU V. 2007 - The Aurignacian in the Zagros region : new research at Yafteh Cave, Lorestan, Iran, Antiquity, 81, p. 82-96.

OTTE M., KOZLOWSKI J.K. 2009 - Origines du Paléolithique supérieur en Asie occidentale, In Le Paléolithique d'Iran, M. Otte, M. Bigliari, J. Jaubert éds., Actes du XV congrès de l'UISPP, Lisbonne, 4-6 septembre 2006, BAR International Series, 1968, p. 57-72.

PESESSE D. 2008a - Les premières sociétés gravettiennes. Analyse comparée des systèmes lithiques de la fin de l'Aurignacien aux débuts du Gravettien. Mémoire de Doctorat, Université de Provence, 2 vol., 276 p., 179 pl.

PESESSE D. 2008b - La Grotte Bouyssonie, première année de fouille, Stratigraphie du Paléolithique supérieur et du Néolithique avec les contributions de J. Chrzavzez, M. Langlais, A. Lenoble, T. Perrin, H. Plisson, J. Primault, Rapport de fouille, SRA Limousin, $103 \mathrm{p}$.

PESESSE D. 2008c - Place du Bayacien dans la structuration du Gravettien. Gallia, t. 50, p. 23-44.

PESESSE D. 2008d - Le statut de la fléchette au sein des premières industries gravettiennes. In J.-Ph. Rigaud (dir.), Entités régionales d'une Paléoculture européenne : Le Gravettien, Actes du colloque, Les Eyzies-de-Tayac, 2004, Paleo, 20, p. 45-58.
PESESSE D. 2010 - Quelques repères pour mieux comprendre l'émergence du Gravettien en France, Bulletin de la Société Préhistorique Française, 105, 3, p. 465-487.

PESESSE D., MICHEL A. 2006 - Le burin des Vachons : apports d'une relecture technologique à la compréhension de l'Aurignacien récent du Nord de l'Aquitaine et des Charentes, Paléo, n 18, p. 143-160.

PEYRONY D. 1946 - Une mise au point au sujet de l'Aurignacien et du Périgordien, Bulletin de la Société Préhistorique Française, t. 42-43, p. 232-237.

PRADEL L. 1952 - Caractéristiques lithiques des différents niveaux périgordiens en France, Bulletin de la Société Préhistorique Française, t. 49, p. 531-543.

PRADEL L. 1978 - La pointe de Font-Yves, Bulletin de la Société Préhistorique Française, t. 75, p. 233-236.

RAYNAL J.-P. 1975 - Recherches sur les dépôts quaternaires des grottes et abris du Bassin permo-triasique de Brive, Mémoire de Doctorat de $3^{\text {e }}$ cycle, Université de Bordeaux I, 2 t.

SCHWABEDISSEN H. 1954 - Die Federmesser-Gruppen des nordwesteuropaïschen Flachlandes. Zur Ausbreitung des Spät-Magdalénien. Karl Wacholtz Verlag, Neumünster.

SONNEVILLE-BORDES D. (de) 1958 - Problèmes généraux du Paléolithique supérieur dans le Sud-ouest de la France, L'Anthropologie, t. 62, p. 413-451.

SONNEVILLE-BORDES D. (de) 1960 - Le Paléolithique supérieur en Périgord, Bordeaux, Delmas, 2 vol., 558 p.

SONNEVILLE BORDES D. (de) PERROT J. 1956 - Lexique typologique du Paléolithique supérieur. Outillage lithique. Bulletin de la Société Préhistorique Française, t. 53, 1956, p. 547-559.

STROBL J., OBERMAIER H. 1909 - Die Aurignacienstation von Krems (N.-Ö.). Jahrbuch für Altertumskunde 3, 1909, 129-148.

TSANOVA T. 2008 - Les débuts du Paléolithique supérieur dans l'Est des Balkans. Réflexion à partir de l'étude taphonomique et techno-économique des ensembles lithiques des sites de Bacho Kiro (couche 11), Temnata (couches VI et 4) et Kozarnika (niveau VII). BAR International Series ; 1752 - 1 vol, 325 p. 